\title{
Simulated tempering with irreversible Gibbs sampling techniques
}

Cite as: J. Chem. Phys. 153, 214111 (2020); https://doi.org/10.1063/5.0025775

Submitted: 18 August 2020 . Accepted: 01 November 2020 . Published Online: 04 December 2020

Fahim Faizi, (D) Pedro J. Buigues, George Deligiannidis, and (D) Edina Rosta

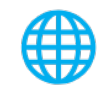

\section{ARTICLES YOU MAY BE INTERESTED IN}

Coarse-grained conformational surface hopping: Methodology and transferability The Journal of Chemical Physics 153, 214110 (2020); https://doi.org/10.1063/5.0031249

Artificial neural networks for predicting charge transfer coupling

The Journal of Chemical Physics 153, 214113 (2020); https://doi.org/10.1063/5.0023697

The challenge of stochastic St $\varnothing$ rmer-Verlet thermostats generating correct statistics The Journal of Chemical Physics 153, 134101 (2020); https://doi.org/10.1063/5.0018962

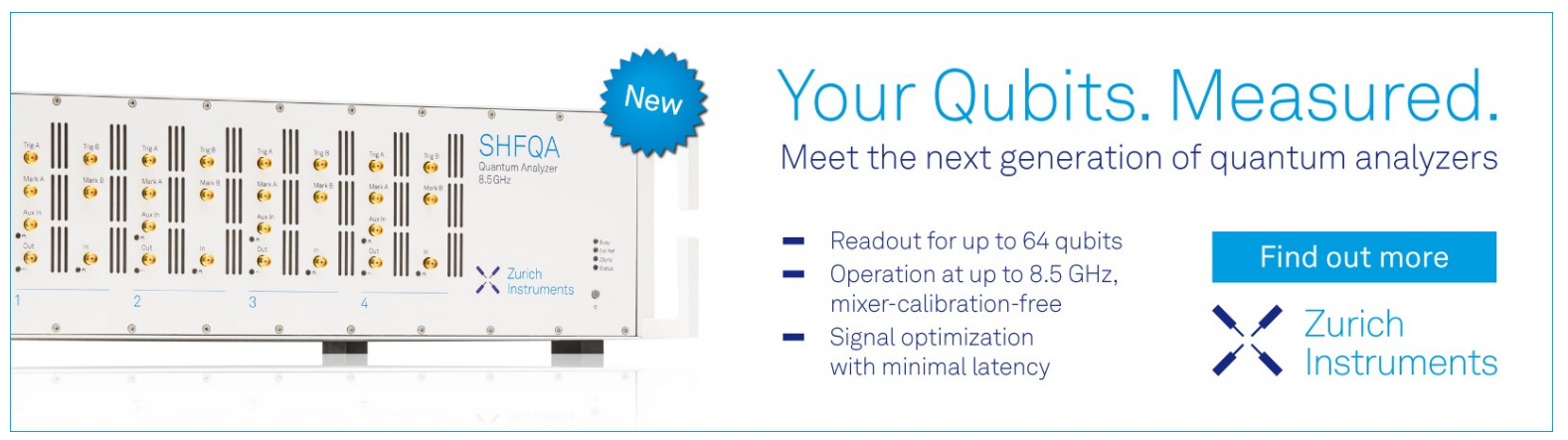




\title{
Simulated tempering with irreversible Gibbs sampling techniques
}

\author{
Cite as: J. Chem. Phys. 153, 214111 (2020); doi: 10.1063/5.0025775 \\ Submitted: 18 August 2020 - Accepted: 1 November 2020 • \\ Published Online: 4 December 2020

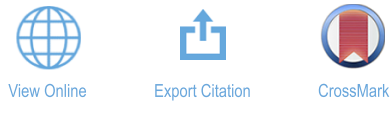

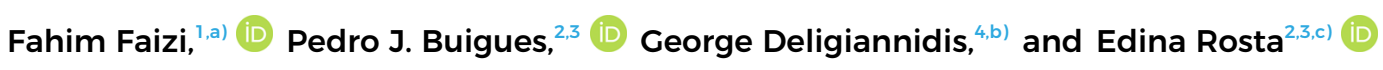

\begin{abstract}
AFFILIATIONS
${ }^{1}$ Department of Mathematics, King's College London, Strand, WC2R 2LS London, United Kingdom

${ }^{2}$ Department of Chemistry, King's College London, 7 Trinity Street, SE1 1DB London, United Kingdom

${ }^{3}$ Department of Physics and Astronomy, University College London, Gower St., Bloomsbury, WC1E 6BT London,

United Kingdom

«Department of Statistics, University of Oxford, 24-29 St Giles', OX1 3LB Oxford, United Kingdom
\end{abstract}

a)Electronic mail: fahim.faizi@kcl.ac.uk

b) Electronic mail: deligian@stats.ox.ac.uk

${ }^{c}$ Author to whom correspondence should be addressed: e.rosta@ucl.ac.uk

\begin{abstract}
We present here two novel algorithms for simulated tempering simulations, which break the detailed balance condition (DBC) but satisfy the skewed detailed balance to ensure invariance of the target distribution. The irreversible methods we present here are based on Gibbs sampling and concern breaking DBC at the update scheme of the temperature swaps. We utilize three systems as a test bed for our methods: a Markov chain Monte Carlo simulation on a simple system described by a one-dimensional double well potential, the Ising model, and molecular dynamics simulations on alanine pentapeptide (ALA5). The relaxation times of inverse temperature, magnetic susceptibility, and energy density for the Ising model indicate clear gains in sampling efficiency over conventional Gibbs sampling techniques with DBC and also over the conventionally used simulated tempering with the Metropolis-Hastings (MH) scheme. Simulations on ALA5 with a large number of temperatures indicate distinct gains in mixing times for inverse temperature and consequently the energy of the system compared to conventional $\mathrm{MH}$. With no additional computational overhead, our methods were found to be more efficient alternatives to the conventionally used simulated tempering methods with DBC. Our algorithms should be particularly advantageous in simulations of large systems with many temperature ladders, as our algorithms showed a more favorable constant scaling in Ising spin systems as compared with both reversible and irreversible $\mathrm{MH}$ algorithms. In future applications, our irreversible methods can also be easily tailored to utilize a given dynamical variable other than temperature to flatten rugged free energy landscapes.
\end{abstract}

Published under license by AIP Publishing. https://doi.org/10.1063/5.0025775

\section{INTRODUCTION}

Algorithms based on Markov Chain Monte Carlo (MCMC) techniques are the most commonly used in Monte Carlo (MC) simulations. The broadly applicable Metropolis-Hastings ( $\mathrm{MH}$ ) algorithm ${ }^{1,2}$ has been implemented in various fields including physics, chemical and biological sciences, ${ }^{5,6}$ and economics. In most cases, one is interested in sampling from intractable multi-dimensional probability distributions with the intention to estimate the expectation value of an observable with respect to the given distribution.
However, when we consider the simulation of complex physical systems, we often find that it remains difficult to efficiently sample them from a target distribution with conventional MCMC algorithms such as the Metropolis-Hastings ${ }^{1,2}(\mathrm{MH})$ and the Gibbs sampler. ${ }^{8}$ Particularly, systems with multiple minimum energy states, such as biopolymers and spin glasses, can often get trapped in local minima.

Extended ensemble MCMC techniques ${ }^{9}$ such as multicanonical methods, ${ }^{10,11}$ the closely related transition matrix Monte Carlo methods, ${ }^{78-80}$ and simulated ${ }^{12}$ and parallel tempering ${ }^{14-17}$ 
provide a solution to explore the state space more efficiently than it is possible with conventional MCMC methods (see also the work of Swendsen and Wang ${ }^{13}$ for an independently developed replica Monte Carlo technique for spin glasses). In this paper, we focus on the simulated tempering method. ${ }^{12}$ In simulated tempering, unlike in conventional methods, the temperature in the Gibbs-Boltzmann distribution is also treated as a dynamical variable along with the configuration. A single replica of the system is therefore simulated with conventional MCMC or Molecular Dynamics (MD), while a temperature change is attempted periodically from among a predetermined discrete set of values. Indeed, at higher temperatures, free energy barriers are lower, and it is therefore more probable that at a higher temperature, the system may cross a free energy barrier and then, upon cooling off again, visit a different energy minimum. The exploration of the temperature space therefore allows the system to escape local minimum energy states by simply transitioning to higher temperatures, and this allows broad sampling of the state space at high temperatures and thorough sampling around local energy minima at low temperatures.

Convergence to the correct enlarged target distribution and therefore invariance at all chosen temperatures can be ensured by a careful construction of the transition rate for temperature change. The most commonly used criterion for temperature change is the $\mathrm{MH}$ algorithm, ${ }^{2}$ which ensures invariance through the detailed balance condition (DBC). However, the DBC is not a strict requirement for invariance. ${ }^{18-20}$ Several studies have shown that breaking it enhances sampling efficiency ${ }^{21-27,29-32}$ and may speed up convergence to the target distribution. ${ }^{33,34}$ The lifting framework, ${ }^{21}$ which violates DBC, has been implemented for several systems. ${ }^{23,25-27,29-32}$ One of the earlier applications of the lifting technique to onedimensional (1D) random walk showed a square root reduction in the mixing time, ${ }^{21}$ which may be an optimal improvement through the lifting framework. ${ }^{22}$ In simulated tempering, ${ }^{12}$ the dynamics of the system in temperature space with $K$ predetermined temperatures can be comparable to a random walk on the one-dimensional lattice with $K$ sites. In this light, Sakai and Hukushima have implemented the lifting framework with the skewed detailed balance condition (SDBC) to the update scheme of the inverse temperature ${ }^{30}$ and have demonstrated (with the Ising model as a test system) a considerable improvement in the relaxation dynamics of the inverse temperature compared to the standard updating scheme of $\mathrm{MH}$ with DBC.

In simulated tempering, the temperature update scheme with the Gibbs sampler (GS) ${ }^{8}$ and its variant, the Metropolized Gibbs sampler (MGS), ${ }^{35}$ has been suggested in some studies. ${ }^{37-39}$ The transition rates for both GS and MGS satisfy the strict DBC; however, we recently proposed their irreversible counterparts with SDBC, namely, the irreversible Gibbs sampler (IGS) and the irreversible Metropolized-Gibbs sampler (IMGS), respectively. ${ }^{36}$ In this paper, we implement IGS and IMGS to the update scheme of inverse temperature in simulated tempering. We apply our simulated tempering methods to three test systems: MCMC simulations on a simple system described by a 1D double well potential and the Ising model and MD simulations on alanine pentapeptide (ALA5). Applications to the Ising model show that the update scheme of inverse temperature $\beta$ with IGS and IMGS can improve the relaxation dynamics of $\beta$ when compared to their respective reversible counterparts with the DBC. Furthermore, the gain in relaxation dynamics of $\beta$ gets exceedingly better with increasing domain size $K$ (i.e., the number of temperatures within a fixed range) when compared to both the conventionally used $\mathrm{MH}$ algorithm and irreversible Metropolis-Hastings (IMH) with SDBC, as implemented by Sakai and Hukushima. ${ }^{30}$ We further demonstrate that both IGS and IMGS reduce the integrated autocorrelation times on magnetic susceptibility and energy density by a considerable factor compared to their reversible counterparts and significantly so compared to both $\mathrm{MH}$ and IMH in large temperature domains. The MD simulations on ALA5 indicate distinct gains in the mixing time of inverse temperature and total energy for a large temperature domain size, but modest gains in the mixing time of the slowest dihedral angles when compared to the conventional simulated tempering with $\mathrm{MH}$.

Assuming a constant specific heat capacity within the range of two temperatures $T_{1}$ and $T_{2}$, the mean energy $\mathscr{E}$ of a system can be assumed to scale as $\mathscr{E} \sim \mathscr{N} k_{B} T$ with the degrees of freedom $\mathscr{N}^{53}$ The difference in mean energy $\Delta \overline{\mathscr{E}}=\overline{\mathscr{E}}\left(T_{2}\right)-\overline{\mathscr{E}}\left(T_{1}\right)$ at $T_{1}$ and $T_{2}$ therefore scales as $\Delta \overline{\mathscr{E}} \sim \mathscr{N} k_{B} \Delta T$. In conventional simulated tempering methods with the $\mathrm{MH}$ scheme, for systems with large degrees of freedom, the temperature spacing is therefore required to be small to ensure overlap of energy distributions at $T_{1}$ and $T_{2}$ for reasonable acceptance probability. For simulations of large systems at a fixed temperature range, sampling of the temperature space becomes inefficient with the conventional simulated tempering, as one would expect with random walks in domains of increasing size. With our methods, the mixing time of inverse temperature and system observables is particularly improved in large temperature domain sizes when compared to conventional methods. We argue that our methods can therefore be more efficient alternatives for the simulation of large systems.

The definition of variables and abbreviations are provided in the nomenclature.

\section{THE SIMULATED TEMPERING METHOD}

One is often interested in using MCMC methods to estimate expectation values under probability distributions with very large dimensions. We may consider a physical system with state space $\Omega$. In classical statistical mechanics, the conditional probability of finding the system in a given configuration $\sigma \in \Omega$ is given by the Gibbs-Boltzmann distribution,

$$
\pi(\boldsymbol{\sigma} \mid \beta)=\frac{1}{Z(\beta)} e^{-\beta H(\boldsymbol{\sigma})},
$$

where $Z(\beta)=\sum_{\Omega} e^{-\beta H(\sigma)}$ is the partition function for a given inverse temperature $\beta$ and $H(\sigma)$ is the Hamiltonian of the system. In conventional MCMC methods, such as the Metropolis-Hastings algorithm, configurations are sampled from the Gibbs-Boltzmann distribution at fixed $\beta$. However, in simulated tempering, $\beta$ is allowed to vary from among a predetermined set of $K$ discrete values $\beta \in\left\{\beta_{1}, \ldots, \beta_{K}\right\}$. In simulated tempering, both $\beta$ and $\sigma \in \Omega$ are therefore stochastic variables. The original state space is enlarged to $\bar{\Omega}:=\Omega \times\{1, \ldots, K\}$ and the probability of finding the system in a given state $\left(\sigma, \beta_{k}\right) \in \bar{\Omega}$ 
is given by the joint probability,

$$
\pi\left(\boldsymbol{\sigma}, \beta_{k}\right)=\frac{1}{\mathscr{Z}} e^{-\beta_{k} H(\boldsymbol{\sigma})+w_{k}},
$$

where the functions $w_{k}=w\left(\beta_{k}\right)$ for $k=1, \ldots, K$ are the weighting factors determined so that the marginal probability distribution, denoted by the probability vector $\pi(\beta)$ $=\left(\pi\left(\beta_{1}\right), \pi\left(\beta_{2}\right), \ldots, \pi\left(\beta_{K}\right)\right)$, is uniform in $\beta$. We will demonstrate this in Eq. (5) shortly. In simulated tempering, in the update scheme of inverse temperature at fixed $\sigma$, we therefore wish to sample from the target probability distribution

$$
\overline{\boldsymbol{\pi}}=\left(\pi\left(\beta_{1} \mid \boldsymbol{\sigma}\right), \pi\left(\beta_{2} \mid \boldsymbol{\sigma}\right), \ldots, \pi\left(\beta_{K} \mid \boldsymbol{\sigma}\right)\right) \quad \forall \boldsymbol{\sigma} \in \Omega,
$$

where $\bar{\pi}$ is a probability vector so that $\pi\left(\beta_{k} \mid \boldsymbol{\sigma}\right)>0$ and $\sum_{k=1}^{K} \pi\left(\beta_{k} \mid \boldsymbol{\sigma}\right)=1$. In essence, simulated tempering therefore involves alternately sampling from the two conditional distributions $\pi(\boldsymbol{\sigma} \mid \beta)$ and $\pi(\beta \mid \boldsymbol{\sigma})$. The generalized partition function $\mathscr{Z}$ is given by

$$
\begin{aligned}
\mathscr{Z} & =\sum_{\bar{\Omega}} e^{-\beta_{k} H(\boldsymbol{\sigma})+w_{k}} \\
& =\sum_{k} \sum_{\Omega} e^{-\beta_{k} H(\boldsymbol{\sigma})+w_{k}} \\
& =\sum_{k} Z\left(\beta_{k}\right) e^{w_{k}} .
\end{aligned}
$$

From Eq. (4), we notice that the partition functions $Z\left(\beta_{k}\right)$ are weighted differently for the given temperatures, where $e^{w_{k}}$ indicates the weight corresponding to the $k$ th temperature and $w_{k}$, the corresponding logarithmic weight. In this paper, we will refer to $w_{k}$ as simply the weights. In simulated tempering, one wishes to avoid confinement of the system in a subspace of the temperature space; therefore, typically a uniform sampling of the temperature space is desired. The determination of the weighs $w_{k}$ is therefore dictated by the requirement that the probability distribution of temperature is flat. This is ideally achieved by setting $w_{k}=-\ln Z\left(\beta_{k}\right)$, the case in which the marginal probability $\pi\left(\beta_{k}\right)$ for a given $\beta_{k}$ becomes constant,

$$
\begin{aligned}
\pi\left(\beta_{k}\right) & =\sum_{\Omega} \pi\left(\sigma, \beta_{k}\right) \\
& =\frac{Z\left(\beta_{k}\right)}{\mathscr{Z}} e^{w_{k}} \\
& =\frac{1}{K} .
\end{aligned}
$$

Notice that $w_{k}=-\ln Z\left(\beta_{k}\right)$ is proportional to the Helmholtz free energy $F$ of the system at $\beta_{k}$, which is given by $\beta_{k} F=-\ln Z\left(\beta_{k}\right)$. The determination of the free energies and therefore of the weights $w_{k}$ is generally difficult to achieve for large complex systems. However, even if the weights are estimated approximately using one of the several iterative methods, ${ }^{40-45}$ a uniform sampling of the temperature space can be realized to a good approximation.

In Algorithm 1, we give a general execution of the simulated tempering method where we have used the notation $X^{(t, \tau)}$ as a state of enlarged state space $\bar{\Omega}$ after $t$ MC-steps of the $\beta$ update and $\tau$ $\mathrm{MC} / \mathrm{MD}$-steps of the $\boldsymbol{\sigma}$ update. $T\left(\boldsymbol{\sigma}^{\prime}, \beta_{l} \mid \boldsymbol{\sigma}, \beta_{k}\right)$ denotes the transition probability from state $\left(\sigma, \beta_{k}\right) \in \bar{\Omega}$ to $\left(\boldsymbol{\sigma}^{\prime}, \beta_{l}\right) \in \bar{\Omega}$. Once the weights are estimated by either short trial simulations (see Ref. 43) or continually adjusted throughout the main simulation (see Ref. 44), a simulated tempering simulation is then executed by alternately performing MC or MD simulations at a fixed $\beta$ [i.e., sampling from the conditional distribution $\pi(\sigma \mid \beta)$ at step 3] and a Monte Carlo step to update $\beta$ at fixed $\sigma$ [that is, sampling from the conditional distribution $\pi(\beta \mid \boldsymbol{\sigma})$ at step 5]. The focus of this paper is on transition probabilities for updating $\beta$ at a fixed configuration $\sigma: T\left(\sigma, \beta_{l} \mid \sigma, \beta_{k}\right)$. In order to ensure convergence to the correct target distribution in Eq. (2), the transition matrix $T\left(\boldsymbol{\sigma}, \beta_{l} \mid \boldsymbol{\sigma}, \beta_{k}\right)$ must satisfy the balance condition (BC),

$$
\pi\left(\boldsymbol{\sigma}, \beta_{k}\right)=\sum_{l=1}^{K} \pi\left(\boldsymbol{\sigma}, \beta_{l}\right) T\left(\boldsymbol{\sigma}, \beta_{k} \mid \boldsymbol{\sigma}, \beta_{l}\right), \quad \forall\left(\boldsymbol{\sigma}, \beta_{k}\right) \in \bar{\Omega} .
$$

In some conventional simulated tempering methods, the $\mathrm{BC}$ is satisfied through the detailed balance condition (DBC),

$$
\pi\left(\boldsymbol{\sigma}, \beta_{k}\right) T\left(\boldsymbol{\sigma}, \beta_{l} \mid \boldsymbol{\sigma}, \beta_{k}\right)=\pi\left(\boldsymbol{\sigma}, \beta_{l}\right) T\left(\boldsymbol{\sigma}, \beta_{k} \mid \boldsymbol{\sigma}, \beta_{l}\right) .
$$

Markov chains that satisfy DBC are reversible chains, while those that violate $\mathrm{DBC}$ are irreversible chains. Perhaps, the most widely used transition probability for updating $\beta$ is that of the MetropolisHastings criterion, ${ }^{2}$ which we discuss in the Subsection titled The Metropolis-Hastings scheme for updating $\beta$.

\section{ALGORITHM 1. Simulated tempering.}

Input: Initialize $X^{(0,0)}=\left(\boldsymbol{\sigma}, \beta^{(0)}\right)$
1: For $t=0, \ldots, \mathscr{T}-1$
2: For $\tau=0, \ldots, \Gamma-1$
3: Sample from $\pi(\boldsymbol{\sigma} \mid \beta)$ : Perform an MC or MD simulation to
$\quad$ update $X^{(t, \tau)}=\left(\boldsymbol{\sigma}, \beta^{(t)}\right)$ to $X^{(t, \tau+1)}=\left(\boldsymbol{\sigma}^{\prime}, \beta^{(t)}\right), \boldsymbol{\sigma}, \boldsymbol{\sigma}^{\prime} \in \Omega$
4: end for
5: Sample from $\pi(\beta \mid \boldsymbol{\sigma}):$ Assuming $X^{(t, \Gamma)}=\left(\boldsymbol{\sigma}, \beta_{k}\right)$, assign
$\quad X^{(t+1, \Gamma)}=\left(\boldsymbol{\sigma}, \beta_{l}\right), \beta_{l} \in\left\{\beta_{1}, \ldots, \beta_{K}\right\}$ with the transition
probability
\[ T\left(\boldsymbol{\sigma}, \beta_{l} \mid \boldsymbol{\sigma}, \beta_{k}\right) \]
6: $X^{(t+1, \Gamma)}=\left(\boldsymbol{\sigma}, \beta^{(t+1)}\right) \rightarrow X^{(t+1,0)}$.
7: end for




\section{UPDATING INVERSE TEMPERATURE WITH DBC}

\section{The Metropolis-Hastings scheme for updating $\beta$}

In conventional simulated tempering, the Metropolis-Hastings ${ }^{2}$ type of transition probability is often used for updating $\beta$. The Metropolis-Hastings algorithm enforces the detailed balance condition by requiring that the stochastic flow $v\left(\sigma, \beta_{l} \mid \sigma, \beta_{k}\right)=\pi\left(\sigma, \beta_{k}\right) T(\sigma$, $\left.\beta_{l} \mid \boldsymbol{\sigma}, \beta_{k}\right)$ is balanced out by its inverse flow $v\left(\boldsymbol{\sigma}, \beta_{k} \mid \boldsymbol{\sigma}, \beta_{l}\right)=\pi(\boldsymbol{\sigma}$, $\left.\beta_{l}\right) T\left(\sigma, \beta_{k} \mid \boldsymbol{\sigma}, \beta_{l}\right)$. The transition probability $T\left(\boldsymbol{\sigma}, \beta_{l} \mid \boldsymbol{\sigma}, \beta_{k}\right)$ from state $\left(\sigma, \beta_{k}\right)$ to $\left(\sigma, \beta_{l}\right)$ (i.e., for updating $\beta$ at a fixed configuration $\left.\sigma\right)$ can be written as

$$
\begin{gathered}
T\left(\boldsymbol{\sigma}, \beta_{l} \mid \boldsymbol{\sigma}, \beta_{k}\right)=Q\left(\boldsymbol{\sigma}, \beta_{l} \mid \boldsymbol{\sigma}, \beta_{k}\right) A\left(\boldsymbol{\sigma}, \beta_{l} \mid \boldsymbol{\sigma}, \beta_{k}\right) \\
\forall \beta_{l} \neq \beta_{k} \in\left\{\beta_{1}, \ldots, \beta_{K}\right\}, \\
T\left(\boldsymbol{\sigma}, \beta_{k} \mid \boldsymbol{\sigma}, \beta_{k}\right)=1-\sum_{\beta_{l} \neq \beta_{k}} T\left(\boldsymbol{\sigma}, \beta_{l} \mid \boldsymbol{\sigma}, \beta_{k}\right),
\end{gathered}
$$

where $Q\left(\boldsymbol{\sigma}, \beta_{l} \mid \boldsymbol{\sigma}, \beta_{k}\right)$ and $A\left(\boldsymbol{\sigma}, \beta_{l} \mid \boldsymbol{\sigma}, \beta_{k}\right)$ denote the proposal and acceptance probabilities, respectively. Hereafter, we assume that the set of inverse temperatures $\left\{\beta_{1}, \ldots, \beta_{K}\right\}$ are equally spaced and ordered such that $\beta_{1}>\beta_{2}>\cdots>\beta_{K}$. Assuming a symmetric proposal, the $\mathrm{MH}$ acceptance probability $A\left(\boldsymbol{\sigma}, \beta_{l} \mid \boldsymbol{\sigma}, \beta_{k}\right)_{\mathrm{MH}}$ is then given by

$$
\begin{aligned}
A\left(\boldsymbol{\sigma}, \beta_{l} \mid \boldsymbol{\sigma}, \beta_{k}\right)_{\mathrm{MH}} & =\min \left[1, \frac{Q\left(\boldsymbol{\sigma}, \beta_{k} \mid \boldsymbol{\sigma}, \beta_{l}\right) \pi\left(\boldsymbol{\sigma}, \beta_{l}\right)}{Q\left(\boldsymbol{\sigma}, \beta_{l} \mid \boldsymbol{\sigma}, \beta_{k}\right) \pi\left(\boldsymbol{\sigma}, \beta_{k}\right)}\right] \forall \beta_{l} \neq \beta_{k} \\
& =\min \left[1, e^{-\Delta}\right]
\end{aligned}
$$

where $\Delta=\left(\beta_{l}-\beta_{k}\right) H(\sigma)-\left(w_{l}-w_{k}\right)$. We immediately notice that the $\mathrm{MH}$ acceptance probability to transition from $\beta_{k}$ to $\beta_{l}$ reduces for large values of $\beta_{l}-\beta_{k}$. Therefore, in practice, the proposal $\beta_{l}$ is often chosen from among $\left\{\beta_{k-1}, \beta_{k+1}\right\}$ such that $Q\left(\sigma, \beta_{k+1} \mid \sigma, \beta_{k}\right)$ $=Q\left(\boldsymbol{\sigma}, \beta_{k-1} \mid \boldsymbol{\sigma}, \beta_{k}\right)=1 / 2, Q\left(\boldsymbol{\sigma}, \beta_{2} \mid \boldsymbol{\sigma}, \beta_{1}\right)=Q\left(\boldsymbol{\sigma}, \beta_{K-1} \mid \boldsymbol{\sigma}, \beta_{K}\right)=1$, and zero otherwise. Now, to update the inverse temperature with the Metropolis-Hastings transition, in step (5) of Algorithm 1, we simply make use of Eq. (9) with the MH acceptance given in (10). We show this explicitly in Algorithm 2.

\section{ALGORITHM 2. Simulated tempering with Metropolis-Hastings.}

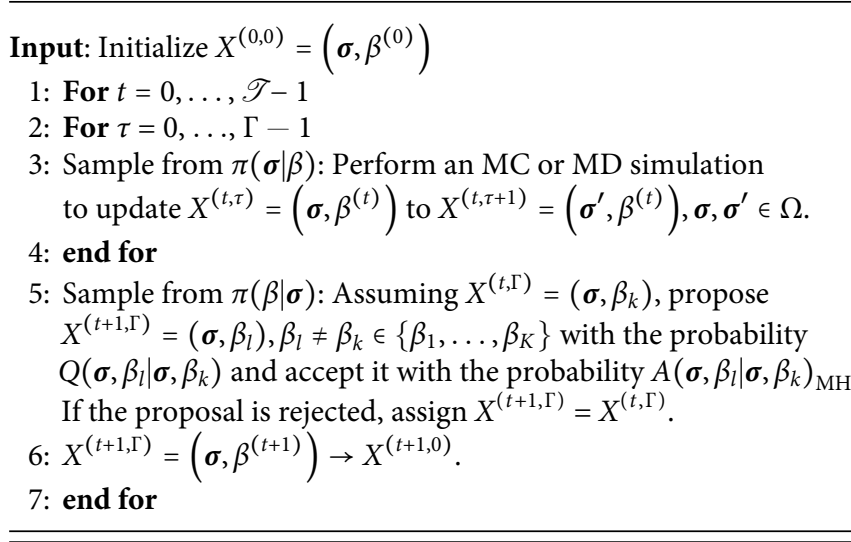

It is a simple exercise to demonstrate that the $\mathrm{MH}$ transition matrix, $T\left(\boldsymbol{\sigma}, \beta_{l} \mid \boldsymbol{\sigma}, \beta_{k}\right)_{\mathrm{MH}}=Q\left(\boldsymbol{\sigma}, \beta_{l} \mid \boldsymbol{\sigma}, \beta_{k}\right) A\left(\boldsymbol{\sigma}, \beta_{l} \mid \boldsymbol{\sigma}, \beta_{k}\right)_{\mathrm{MH}}$, satisfies the DBC in (7) and therefore ensures the invariance of the target distribution.

\section{The Gibbs sampler for updating $\beta$}

In the Gibbs sampler (GS), ${ }^{8}$ also known as the heat-bath algorithm in statistical physics, the inverse temperature is updated, whereby a new $\beta_{l} \in\left\{\beta_{1}, \ldots, \beta_{K}\right\}$ is drawn from its conditional distribution $\pi(\cdot \mid \boldsymbol{\sigma})$. We let $G\left(\boldsymbol{\sigma}, \beta_{l} \mid \boldsymbol{\sigma}, \beta_{k}\right)$ to denote the Gibbs transition probability from $\beta_{k}$ to $\beta_{l}$, which is simply the conditional distribution given $\sigma$,

$$
G\left(\boldsymbol{\sigma}, \beta_{l} \mid \boldsymbol{\sigma}, \beta_{k}\right)=\frac{\pi\left(\boldsymbol{\sigma}, \beta_{l}\right)}{\sum_{r=1}^{K} \pi\left(\boldsymbol{\sigma}, \beta_{r}\right)} \quad \forall\left(\boldsymbol{\sigma}, \beta_{l}\right) \in \bar{\Omega},
$$

where $\pi\left(\boldsymbol{\sigma}, \beta_{l}\right)$ is given in (2). Notice that the Gibbs transition to the new value $\beta_{l}$ is independent of the current value $\beta_{k}$. The execution of simulated tempering with the Gibbs sampler is then straightforward: In step (5) of Algorithm 1, the generic transition probability in (8) is now replaced with the Gibbs transition given in (11). Note that for a fixed configuration $\sigma$, the computational cost of the summation in (11) is next to negligible even for excessively large $K$ values. The Gibbs sampler is, in fact, a special case of the Metropolis-Hastings algorithm whereby every proposal is accepted. This is easily demonstrated by letting the proposal $Q\left(\boldsymbol{\sigma}, \beta_{l} \mid \boldsymbol{\sigma}, \beta_{k}\right)=\pi\left(\beta_{l} \mid \boldsymbol{\sigma}\right)$, the case in which the $\mathrm{MH}$ acceptance probability for every proposal is then exactly one,

$$
A\left(\boldsymbol{\sigma}, \beta_{l} \mid \boldsymbol{\sigma}, \beta_{k}\right)=\min \left[1, \frac{\pi\left(\beta_{k} \mid \boldsymbol{\sigma}\right) \pi\left(\boldsymbol{\sigma}, \beta_{l}\right)}{\pi\left(\beta_{l} \mid \boldsymbol{\sigma}\right) \pi\left(\boldsymbol{\sigma}, \beta_{k}\right)}\right]=1
$$

As a special case of the Metropolis-Hastings criteria, the Gibbs sampler therefore ensures the invariance of the target distribution. The Metropolis-Hastings acceptance in (10) is dependent on the spacing $\left(\beta_{l}-\beta_{k}\right)$, and the acceptance probabilities for large jumps in temperature space are therefore small. However, the Gibbs transition probability is independent of the current inverse temperature $\beta_{k}$, and it is therefore capable of providing a more efficient global exploration of temperature space than that is possible with the standard Metropolis-Hastings method. Simulated tempering with the Gibbs sampler, whereby the Gibbs transition in (11) is used to update the inverse temperature, has been implemented in several studies ${ }^{37-39}$ that demonstrate better performance compared with the conventional method of updating $\beta$ with the Metropolis-Hastings method. However, for reasons unclear, it is not as widely in practice as the standard Metropolis-Hastings method.

\section{The Metropolized-Gibbs sampler for updating $\beta$}

We now briefly introduce a variant of the Gibbs sampler, namely, the Metropolized-Gibbs sampler (MGS), which was originally introduced by $\mathrm{Liu}^{35}$ as a modification of the random scan Gibbs sampler with an improved mixing rate. The MGS transition matrix, 
which also satisfies DBC, provides an improved sampling of the state space compared to the standard Gibbs transition in (11). The development of the Metropolized-Gibbs sampler was directly motivated by Peskun's theorem: Given that both transition matrices $\left(T_{i j}^{A}\right)_{i, j \in \Omega}$ and $\left(T_{i j}^{B}\right)_{i, j \in \Omega}$ satisfy DBC and $T_{i i}^{A}<T_{i i}^{B} \forall i \in \Omega$, then a Markov chain with the transition matrix $T_{i j}^{A}$ returns estimates with smaller asymptotic variance than a Markov chain with transition matrix $T_{i j}^{B}$. In other words, minimizing the probability of remaining in the current state increases mobility in the state space and therefore provides a more efficient sampling of the state space. In the context of simulated tempering for updating $\beta$, a new candidate, $\beta_{l} \neq \beta_{k}$, is proposed with the probability

$$
Q\left(\boldsymbol{\sigma}, \beta_{l} \mid \boldsymbol{\sigma}, \beta_{k}\right)=\frac{G\left(\boldsymbol{\sigma}, \beta_{l} \mid \boldsymbol{\sigma}, \beta_{k}\right)}{1-G\left(\boldsymbol{\sigma}, \beta_{k} \mid \boldsymbol{\sigma}, \beta_{l}\right)} \quad \forall \beta_{l} \neq \beta_{k} \in\left\{\beta_{1}, \ldots, \beta_{K}\right\}
$$

and accepted with the Metropolis-Hastings acceptance probability [Eq. (10)]

$$
A\left(\boldsymbol{\sigma}, \beta_{l} \mid \boldsymbol{\sigma}, \beta_{k}\right)=\min \left[1, \frac{1-G\left(\boldsymbol{\sigma}, \beta_{k} \mid \boldsymbol{\sigma}, \beta_{l}\right)}{1-G\left(\boldsymbol{\sigma}, \beta_{l} \mid \boldsymbol{\sigma}, \beta_{k}\right)}\right] \quad \forall \beta_{l} \neq \beta_{k},
$$

where, upon rejection, the current state $\left(\sigma, \beta_{k}\right)$ is retained. The reversible transition matrix $M\left(\sigma, \beta_{l} \mid \boldsymbol{\sigma}, \beta_{k}\right)$ for the MetropolizedGibbs sampler can then be written as

$$
\begin{aligned}
M\left(\boldsymbol{\sigma}, \beta_{l} \mid \boldsymbol{\sigma}, \beta_{k}\right)= & \min \left[\frac{G\left(\boldsymbol{\sigma}, \beta_{l} \mid \boldsymbol{\sigma}, \beta_{k}\right)}{1-G\left(\boldsymbol{\sigma}, \beta_{k} \mid \boldsymbol{\sigma}, \beta_{l}\right)}, \frac{G\left(\boldsymbol{\sigma}, \beta_{l} \mid \boldsymbol{\sigma}, \beta_{k}\right)}{1-G\left(\boldsymbol{\sigma}, \beta_{l} \mid \boldsymbol{\sigma}, \beta_{k}\right)}\right] \\
& \forall \beta_{l} \neq \beta_{k} \in\left\{\beta_{1}, \ldots, \beta_{K}\right\}, \\
M\left(\boldsymbol{\sigma}, \beta_{k} \mid \boldsymbol{\sigma}, \beta_{k}\right)= & 1-\sum_{\beta_{l} \neq \beta_{k}} M\left(\boldsymbol{\sigma}, \beta_{l} \mid \boldsymbol{\sigma}, \beta_{k}\right),
\end{aligned}
$$

which satisfies DBC. A few points here merit some elaboration; we point out that for a two state solution, $K=2$, The Gibbs transition probability in (11) becomes equivalent to Barker's method, whereas the MGS transition in (15) decomposes to the standard Metropolis-Hastings transition. Peskun had demonstrated that, within DBC, the Metropolis-Hastings criterion is superior to Barker's method as the former returns a smaller probability of remaining in the current state and therefore increases mobility in the state space. This argument applies more generally to the MGS sampler. By minimizing the probability of retaining the current state, the MGS transition, $M\left(\sigma, \beta_{l} \mid \boldsymbol{\sigma}, \beta_{k}\right)$, is more efficient at sampling of the state space than the Gibbs transition $G\left(\sigma, \beta_{l} \mid \sigma, \beta_{k}\right)$. This has been numerically demonstrated in some studies. ${ }^{36,38,47}$ In practice, to update the inverse temperature with the MGS sampler, one simply replaces the generic transition probability in (8) with that of the MGS transition given in (15).

\section{UPDATING INVERSE TEMPERATURE WITH SDBC}

\section{The lifting framework}

In the lifting framework, as introduced by Diaconis et al. ${ }^{21}$ the state space is enlarged by effectively replicating a duplicate copy of the original space. Each replica, which is characterized by a lifting variable $\varepsilon \in\{-1,+1\}$, consists of all configurations $\sigma \in \Omega$ as in the original space. The system now explores an extended state space, that is, in addition to the intra-replica transition between configurations $\sigma \rightarrow \sigma^{\prime}$ as in the original space, the system can now also perform inter-replica transition $(\sigma, \varepsilon) \rightarrow(\sigma,-\varepsilon)$ between duplicate copies of a given configuration. A Markov chain propagated in this enlarged state space breaks the DBC but ensures convergence to the target distribution by satisfying BC. . $^{21,25,26,29,36}$ In this section, we utilize the lifting framework with SDBC, as proposed by Turitsyn et al. ${ }^{25}$ In particular, we implement the lifting framework in the updating scheme of inverse temperature in simulated tempering. Sakai and Hukushima ${ }^{30}$ have already implemented lifting with SDBC to the Metropolis-Hastings transition $T\left(\boldsymbol{\sigma}, \beta_{l} \mid \boldsymbol{\sigma}, \beta_{k}\right)_{\mathrm{MH}}$ $=Q\left(\boldsymbol{\sigma}, \beta_{l} \mid \boldsymbol{\sigma}, \beta_{k}\right) A\left(\boldsymbol{\sigma}, \beta_{l} \mid \boldsymbol{\sigma}, \beta_{k}\right)_{\mathrm{MH}}$ for updating $\beta$. The authors applied their algorithm to the simulation of the $2 \mathrm{D}$ Ising model and demonstrated that, when compared with the conventional MetropolisHastings method with the DBC, their algorithm provides significant improvement in the relaxation dynamics of $\beta$ and the magnetization of the model. Hereafter, we will refer to the algorithm of Sakai and Hukushima $^{30}$ as irreversible Metropolis-Hastings (IMH). We had recently proposed the irreversible counterparts of the Gibbs sampler and the Metropolized-Gibbs sampler, namely, IGS and IMGS where both satisfy the SDBC. ${ }^{36}$ Here, we demonstrate that both IGS and IMGS can be adapted for the update scheme of inverse temperature. Numerical simulations in the next section show that, when compared with their respective reversible counterparts, both IGS and IMGS improve the relaxation dynamics of $\beta$ and consequently that of some system observables. Furthermore, our results also show considerable improvement over the IMH algorithm in the mixing time of $\beta$ and that of some system observables for large temperature domains $K$.

\section{SDBC in the context of simulated tempering}

The lifting variable $\varepsilon \in\{+1,-1\}$ is introduced to double the state space $\bar{\Omega}$ so that the extended state space $\tilde{\Omega}:=\bar{\Omega} \times\{+1,-1\}$ now consist of two replicas characterized by $\varepsilon= \pm$. The probability of finding the system in state $\left(\sigma, \beta_{k}, \varepsilon\right)$ is given by

$$
\tilde{\pi}\left(\sigma, \beta_{k}, \varepsilon\right)=\frac{1}{2} \pi\left(\sigma, \beta_{k}\right)
$$

To update $\beta$, we now wish to sample from the extended target distribution given by the probability vector

$$
\begin{aligned}
\tilde{\boldsymbol{\pi}} & =\left(\tilde{\pi}\left(\beta_{1} \mid \boldsymbol{\sigma},+\right), \ldots, \tilde{\pi}\left(\beta_{K} \mid \boldsymbol{\sigma},+\right), \tilde{\pi}\left(\beta_{1} \mid \boldsymbol{\sigma},-\right), \ldots, \tilde{\pi}\left(\beta_{K} \mid \boldsymbol{\sigma},-\right)\right) \\
& =\frac{1}{2}(\overline{\boldsymbol{\pi}}, \tilde{\boldsymbol{\pi}})
\end{aligned}
$$

where the original target $\bar{\pi}$ is given in (3). Notably, $\tilde{\pi}\left(\boldsymbol{\sigma}, \beta_{k}, \varepsilon\right)$ $=\tilde{\pi}\left(\boldsymbol{\sigma}, \beta_{k},-\varepsilon\right)$ and the marginal $\sum_{\varepsilon^{\prime}} \tilde{\pi}\left(\boldsymbol{\sigma}, \beta_{k}, \varepsilon^{\prime}\right)=\pi\left(\boldsymbol{\sigma}, \beta_{k}\right)$. The transition matrix $\tilde{\boldsymbol{T}}$ of the Markov chain on the extended state space $\tilde{\Omega}$ is now given by 


$$
\widetilde{\boldsymbol{T}}=\left(\begin{array}{cc}
T\left(\boldsymbol{\sigma}, \beta_{l},+\mid \boldsymbol{\sigma}, \beta_{k},+\right) & \Lambda\left(\boldsymbol{\sigma}, \beta_{k},-\mid \boldsymbol{\sigma}, \beta_{k},+\right) \\
\Lambda\left(\boldsymbol{\sigma}, \beta_{k},+\mid \boldsymbol{\sigma}, \beta_{k},-\right) & T\left(\boldsymbol{\sigma}, \beta_{l},-\mid \boldsymbol{\sigma}, \beta_{k},-\right)
\end{array}\right)
$$

where $T\left(\boldsymbol{\sigma}, \beta_{l}, \pm \mid \boldsymbol{\sigma}, \beta_{k}, \pm\right) \geq 0$ denotes the intra-replica transition probability from state $\left(\sigma, \beta_{k}\right) \in \bar{\Omega}$ to $\left(\sigma, \beta_{l}\right) \in \bar{\Omega}$ in the respective $\varepsilon= \pm$ replicas. The inter-replica transition probability from state $\left(\boldsymbol{\sigma}, \beta_{k}, \pm\right)$ to $\left(\boldsymbol{\sigma}, \beta_{k}, \mp\right)$ is denoted by $\Lambda\left(\boldsymbol{\sigma}, \beta_{k}, \mp \mid \boldsymbol{\sigma}, \beta_{k}, \pm\right) \geq 0$. Note that $\Lambda\left(\boldsymbol{\sigma}, \beta_{l}, \mp \mid \boldsymbol{\sigma}, \beta_{k}, \pm\right)=0 \forall \beta_{l} \neq \beta_{k} \in\left\{\beta_{1}, \ldots, \beta_{K}\right\}$. The normalization of probability in the extended state space can be written as

$$
\sum_{l=1}^{K} T\left(\boldsymbol{\sigma}, \beta_{l}, \varepsilon \mid \boldsymbol{\sigma}, \beta_{k}, \varepsilon\right)+\Lambda\left(\boldsymbol{\sigma}, \beta_{k},-\varepsilon \mid \boldsymbol{\sigma}, \beta_{k}, \varepsilon\right)=1 .
$$

Assuming that the transition matrix $\widetilde{T}$ is ergodic, it must then satisfy the BC $\tilde{\pi}=\tilde{\pi} \widetilde{T}$ to ensure invariance of the target distribution $\tilde{\pi}$. The $\mathrm{BC}$ takes the explicit form

$$
\begin{array}{rl}
\sum_{l=1}^{K} & T\left(\boldsymbol{\sigma}, \beta_{l}, \varepsilon \mid \boldsymbol{\sigma}, \beta_{k}, \varepsilon\right) \tilde{\pi}\left(\boldsymbol{\sigma}, \beta_{k}, \varepsilon\right)+\Lambda\left(\boldsymbol{\sigma}, \beta_{k},-\varepsilon \mid \boldsymbol{\sigma}, \beta_{k}, \varepsilon\right) \tilde{\pi}\left(\boldsymbol{\sigma}, \beta_{k}, \varepsilon\right) \\
= & \sum_{l=1}^{K} T\left(\boldsymbol{\sigma}, \beta_{k}, \varepsilon \mid \boldsymbol{\sigma}, \beta_{l}, \varepsilon\right) \tilde{\pi}\left(\boldsymbol{\sigma}, \beta_{l}, \varepsilon\right) \\
& +\Lambda\left(\boldsymbol{\sigma}, \beta_{k}, \varepsilon \mid \boldsymbol{\sigma}, \beta_{k},-\varepsilon\right) \tilde{\pi}\left(\boldsymbol{\sigma}, \beta_{k},-\varepsilon\right),
\end{array}
$$

$\forall \beta_{k} \in\left\{\beta_{1}, \ldots, \beta_{K}\right\}$. The $\mathrm{BC}$ can be satisfied by imposing the skewed detailed balance condition (SDBC) on the transition matrix,

$$
\tilde{\pi}\left(\boldsymbol{\sigma}, \beta_{k}, \varepsilon\right) T\left(\boldsymbol{\sigma}, \beta_{l}, \varepsilon \mid \boldsymbol{\sigma}, \beta_{k}, \varepsilon\right)=\tilde{\pi}\left(\boldsymbol{\sigma}, \beta_{l},-\varepsilon\right) T\left(\boldsymbol{\sigma}, \beta_{k},-\varepsilon \mid \boldsymbol{\sigma}, \beta_{l},-\varepsilon\right) .
$$

The SDBC requires that the stochastic flow from state $\left(\sigma, \beta_{k}\right) \rightarrow\left(\sigma, \beta_{l}\right)$ in one replica is balanced by reverse flow $\left(\sigma, \beta_{l}\right)$ $\rightarrow\left(\sigma, \beta_{k}\right)$ in the other replica. The SDBC therefore by definition breaks the detailed balance condition, $\tilde{\pi}\left(\boldsymbol{\sigma}, \beta_{k}, \varepsilon\right) T\left(\boldsymbol{\sigma}, \beta_{l}, \varepsilon \mid \boldsymbol{\sigma}, \beta_{k}, \varepsilon\right)$ $\neq \tilde{\pi}\left(\boldsymbol{\sigma}, \beta_{l}, \varepsilon\right) T\left(\boldsymbol{\sigma}, \beta_{k}, \varepsilon \mid \boldsymbol{\sigma}, \beta_{l}, \varepsilon\right)$. Note that by imposing SDBC on the transition matrix, we can obtain a condition for the construction of the inter-replica transition probability $\Lambda\left(\boldsymbol{\sigma}, \beta_{k},-\varepsilon \mid \boldsymbol{\sigma}, \beta_{k}, \varepsilon\right)$, and we see this clearly once we insert (21) into (20) to obtain

$$
\begin{aligned}
& \Lambda\left(\boldsymbol{\sigma}, \beta_{k},-\varepsilon \mid \boldsymbol{\sigma}, \beta_{k}, \varepsilon\right)-\Lambda\left(\boldsymbol{\sigma}, \beta_{k}, \varepsilon \mid \boldsymbol{\sigma}, \beta_{k},-\varepsilon\right) \\
& \quad=\sum_{l \neq k}\left[T\left(\boldsymbol{\sigma}, \beta_{l},-\varepsilon \mid \boldsymbol{\sigma}, \beta_{k},-\varepsilon\right)-T\left(\boldsymbol{\sigma}, \beta_{l}, \varepsilon \mid \boldsymbol{\sigma}, \beta_{k}, \varepsilon\right)\right] .
\end{aligned}
$$

A particular solution of (22), which was originally proposed by Turitsyn et al., ${ }^{25}$ is of the form

$$
\begin{aligned}
& \Lambda\left(\boldsymbol{\sigma}, \beta_{k},-\varepsilon \mid \boldsymbol{\sigma}, \beta_{k}, \varepsilon\right) \\
& \quad=\max \left[0, \sum_{l \neq k}\left(T\left(\boldsymbol{\sigma}, \beta_{l},-\varepsilon \mid \boldsymbol{\sigma}, \beta_{k},-\varepsilon\right)-T\left(\boldsymbol{\sigma}, \beta_{l}, \varepsilon \mid \boldsymbol{\sigma}, \beta_{k}, \varepsilon\right)\right)\right],
\end{aligned}
$$

which is known as the Turitsyn-Chertkov-Vucelja (TCV) solution. However, several alternative solutions of (22) have been proposed and studied, ${ }^{29}$ as the alternative choice known as the Sakai-Hukushima 1 (SH1) solution, which is given by

$$
\Lambda\left(\boldsymbol{\sigma}, \beta_{k},-\varepsilon \mid \boldsymbol{\sigma}, \beta_{k}, \varepsilon\right)=\sum_{l \neq k} T\left(\boldsymbol{\sigma}, \beta_{l},-\varepsilon \mid \boldsymbol{\sigma}, \beta_{k},-\varepsilon\right),
$$

has been studied in the context of the 1D Ising model.

Our task at hand is now to construct an intra-replica transition matrix $T\left(\boldsymbol{\sigma}, \beta_{l}, \varepsilon \mid \boldsymbol{\sigma}, \beta_{k}, \varepsilon\right)$ that satisfies the SDBC given in (21). With this in mind, we follow the same procedure we had outlined recently, ${ }^{36}$ which involves modifying a generic transition matrix $T\left(\boldsymbol{\sigma}, \beta_{l} \mid \boldsymbol{\sigma}, \beta_{k}\right)$ that satisfies DBC in (7), with the skewness function $\Theta\left(\beta_{l}, \varepsilon \mid \beta_{k}, \varepsilon\right)$. We therefore define

$$
T\left(\boldsymbol{\sigma}, \beta_{l}, \varepsilon \mid \boldsymbol{\sigma}, \beta_{k}, \varepsilon\right)=\Theta\left(\beta_{l}, \varepsilon \mid \beta_{k}, \varepsilon\right) T\left(\boldsymbol{\sigma}, \beta_{l} \mid \boldsymbol{\sigma}, \beta_{k}\right),
$$

where the skewness function has the properties

$$
0 \leq \Theta\left(\beta_{l}, \varepsilon \mid \beta_{k}, \varepsilon\right) \leq 1
$$

and

$$
\Theta\left(\beta_{l}, \varepsilon \mid \beta_{k}, \varepsilon\right)=\Theta\left(\beta_{k},-\varepsilon \mid \beta_{l},-\varepsilon\right) .
$$

With this definition, we note that the transition matrix $T\left(\boldsymbol{\sigma}, \beta_{l}, \varepsilon \mid \boldsymbol{\sigma}, \beta_{k}, \varepsilon\right)$ in (25) now satisfies the SDBC in (21). This completes our description of the extended transition matrix $\tilde{\boldsymbol{T}}$, as defined in (18). An irreversible Markov chain can therefore be propagated on the extended state space $\tilde{\Omega}$, whereby the stationary distribution of the chain will, by the arguments above, converge to the invariant target distribution. Next, we introduce an explicit form of a suitable skewness function and demonstrate how to adapt the IGS and IMGS $^{36}$ to the update scheme of inverse temperature.

\section{Irreversible Gibbs sampler for updating $\beta$}

In principle, any skewness function that meets conditions (26) and (27) will suffice to construct a transition matrix $T\left(\boldsymbol{\sigma}, \beta_{l}, \varepsilon \mid \boldsymbol{\sigma}, \beta_{k}, \varepsilon\right)$. However, certain choices of the skewness function may lead to more efficient sampling of the temperature space than others. Here, we make use of the skewness function we had recently proposed, ${ }^{36}$ which we express in general formulation in the context of simulated tempering,

$$
\Theta\left(f_{l}, \varepsilon \mid f_{k}, \varepsilon\right)=\varphi(1+\delta \varepsilon \Phi(f))
$$

where $f \in\left\{f_{1}, \ldots, f_{K}\right\}$ is the lifting coordinate and the function $\Phi(f)=\operatorname{sgn}\left(f_{l}-f_{k}\right)$ with the sign function given by

$$
\operatorname{sgn}(x)=\left\{\begin{array}{cc}
-1 & \text { if } x<0 \\
0 & \text { if } x=0 \\
+1 & \text { if } x>0
\end{array}\right.
$$

The constant $\varphi=1 /(1+\delta)$. The deviation parameter $\delta \in[0,1]$ determines the extent to which the $\mathrm{DBC}$ is violated, and the $\mathrm{DBC}$ 
is recovered by setting $\delta=0$. Notably, with $\delta=0$, the transition $T\left(\boldsymbol{\sigma}, \beta_{l}, \varepsilon \mid \boldsymbol{\sigma}, \beta_{k}, \varepsilon\right)$ in (25) becomes uniform in $\varepsilon$, and consequently, the SDBC in (21) reduces to the DBC in (7).

In general applications of the lifting framework with the SDBC, the lifting coordinate $f$ is often taken to be an observable of interest, as in the context of spin systems, this could be the magnetization $^{25-28,36}$ or energy of the system. ${ }^{26,36}$ Often $f$ is assigned to be an observable with slow relaxation dynamics so that by lifting it, a more efficient sampling of the state space can be realized along the coordinates of this particular observable. In temperature simulated tempering, particularly in the update scheme of $\beta$, one is often interested in improving the mixing rate in temperature space. We therefore assign the lifting coordinate $f$ to be the inverse temperature $\beta$. However, considering a simulated tempering equivalent of Hamiltonian replica exchange, ${ }^{53}$ one may assign a dynamical variable other than $\beta$ as the lifting coordinate, such as an interaction parameter (e.g., different strengths of an external magnetic field) in the Hamiltonian of spin systems.

The skewness function in (28) effectively introduces a bias in the way the variable $f$ is sampled. To better understand this, we set the lifting coordinate $f$ as the inverse temperature and consider two distinct cases: $(\varepsilon= \pm, \Phi(\beta)= \pm)$ and $(\varepsilon= \pm, \Phi(\beta)=\mp)$. The transition probability $T\left(\sigma, \beta_{l}, \varepsilon \mid \sigma, \beta_{k}, \varepsilon\right)$ in (25) then breaks down to $T\left(\boldsymbol{\sigma}, \beta_{l} \mid \boldsymbol{\sigma}, \beta_{k}\right)$ for $(\varepsilon= \pm 1, \Phi(\beta)= \pm 1)$ and $(1-\delta / 1+\delta) T\left(\boldsymbol{\sigma}, \beta_{l} \mid \boldsymbol{\sigma}, \beta_{k}\right)$ for $(\varepsilon= \pm 1, \Phi(\beta)=\mp 1)$. Considering the $\varepsilon=+1$ replica and $\delta \neq 0$ as an example, we observe that Monte Carlo moves that increase $\beta$ [i.e., $\Phi(\beta)=+1$ ] are stochastically favored over moves that decrease $\beta$ [i.e., $\Phi(\beta)=-1$ ], while the opposite is true in the $\varepsilon=-1$ replica. In the two replicas, the system therefore stochastically favors opposing and fixed directions in temperature space.

Notably, inserting the Metropolis-Hastings transition $T\left(\boldsymbol{\sigma}, \beta_{l} \mid \boldsymbol{\sigma}, \beta_{k}\right)_{\mathrm{MH}}$ on the right-hand side of (25) (also setting $\varphi=1$ ) leads to the IMH algorithm of Sakai and Hukushima. ${ }^{30}$ The IMH algorithm, in particular, introduces a bias in the proposal $Q(\sigma$, $\left.\beta_{l} \mid \boldsymbol{\sigma}, \beta_{k}\right)$ in the nearest neighbor exchange scheme for $\beta$. In this scheme, the dynamical behavior of a fixed configuration $\sigma$ is comparable to a simple random walk in temperature space with $K$ states. Sakai and Hukushima have numerically demonstrated with the Ising model that the IMH algorithm suppresses the diffusive behavior of $\sigma$ on the temperature space so that the mixing time of $\beta$ now scales on the order of $\mathscr{O}(K)$ with the IMH algorithm as opposed to $\mathscr{O}\left(K^{2}\right)$ with conventional $\mathrm{MH}$ with DBC. However, as we have seen in previous sections, other than the conventional Metropolis-Hastings algorithm, there are several alternative choices with the DBC to sample from the conditional distribution $\pi(\beta \mid \sigma)$. We argue here that the irreversible counterparts of the Gibbs sampler and Metropolized-Gibbs sampler can be constructed to sample from the conditional $\pi(\beta \mid \sigma)$ through satisfying the SDBC. As we will see in the Section titled Performance analysis with MCMC simulations, the resulting IGS and IMGS schemes for updating $\beta$ provide an improvement in the relaxation dynamics of $\beta$ and system observables over their respective reversible counterparts with the $\mathrm{DBC}$ and also when compared with the IMH algorithm.

We proceed to adapt the irreversible Gibbs sampler ${ }^{36}$ for the update scheme of $\beta$. We do this by inserting the Gibbs transition, $G\left(\boldsymbol{\sigma}, \beta_{l} \mid \boldsymbol{\sigma}, \beta_{k}\right)$ given in (11), on the right-hand side of (25). The IGS intra-replica transition matrix $\mathscr{G}\left(\boldsymbol{\sigma}, \beta_{l}, \varepsilon \mid \boldsymbol{\sigma}, \beta_{k}, \varepsilon\right)$ is then defined as

$$
\begin{aligned}
\mathscr{G}\left(\boldsymbol{\sigma}, \beta_{l}, \varepsilon \mid \boldsymbol{\sigma}, \beta_{k}, \varepsilon\right) & =\Theta\left(\beta_{l}, \varepsilon \mid \beta_{k}, \varepsilon\right) G\left(\boldsymbol{\sigma}, \beta_{l} \mid \boldsymbol{\sigma}, \beta_{k}\right) \\
\forall & \beta_{l} \neq \beta_{k} \in\left\{\beta_{1}, \ldots, \beta_{K}\right\}, \\
\mathscr{G}\left(\boldsymbol{\sigma}, \beta_{k}, \varepsilon \mid \boldsymbol{\sigma}, \beta_{k}, \varepsilon\right) & =1-\sum_{l \neq k} \mathscr{G}\left(\boldsymbol{\sigma}, \beta_{l}, \varepsilon \mid \boldsymbol{\sigma}, \beta_{k}, \varepsilon\right) .
\end{aligned}
$$

We recover the inter-replica transition probability of the TCV solution from the general formulation in (23),

$$
\begin{aligned}
& \Lambda\left(\boldsymbol{\sigma}, \beta_{k},-\varepsilon \mid \boldsymbol{\sigma}, \beta_{k}, \varepsilon\right)_{\mathrm{IGS}} \\
& \quad=\max \left[0, \sum_{l \neq k}\left(\mathscr{G}\left(\boldsymbol{\sigma}, \beta_{l},-\varepsilon \mid \boldsymbol{\sigma}, \beta_{k},-\varepsilon\right)-\mathscr{G}\left(\boldsymbol{\sigma}, \beta_{l}, \varepsilon \mid \boldsymbol{\sigma}, \beta_{k}, \varepsilon\right)\right)\right] .
\end{aligned}
$$

This completes our description of the irreversible Gibbs sampler for the update scheme of inverse temperature in simulated tempering. The general execution of the IGS to update $\beta$ is given in Algorithm 3 where we have used the notation $\tilde{X}^{(t, \tau)}$ as a state of the extended state space $\tilde{\Omega}$ after $t$ MC steps of $\beta$ updates and $\tau$ MC/MD steps of $\sigma$ updates.

The implementation of the irreversible Gibbs sampler as given in Algorithm 3 differs from that of the conventional Gibbs sampler of Eq. (11) with DBC in steps 5-7. Therefore, to verify that the steps shown in Algorithm 3 lead to sampling from the correct target distribution, it suffices to demonstrate that the conditional $\tilde{\pi}(\beta, \varepsilon \mid \boldsymbol{\sigma})=1 / 2 \pi(\beta \mid \boldsymbol{\sigma})$ satisfies the balance condition. We show this in the Appendix.

\section{Irreversible Metropolized-Gibbs sampler for updating $\beta$}

Likewise, we briefly demonstrate that the $\mathrm{IMGS}^{36}$ can be adapted to sample from the conditional distribution $\pi(\beta \mid \sigma)$. We insert the Metropolized-Gibbs transition $M\left(\sigma, \beta_{l} \mid \boldsymbol{\sigma}, \beta_{k}\right)$, as given in (15), on the right-hand side of (25) to obtain the transition matrix $\mathscr{M}\left(\boldsymbol{\sigma}, \beta_{l}, \varepsilon \mid \boldsymbol{\sigma}, \beta_{k}, \varepsilon\right)$ for IMGS,

$$
\begin{gathered}
\mathscr{M}\left(\boldsymbol{\sigma}, \beta_{l}, \varepsilon \mid \boldsymbol{\sigma}, \beta_{k}, \varepsilon\right)=\Theta\left(\beta_{l}, \varepsilon \mid \beta_{k}, \varepsilon\right) M\left(\boldsymbol{\sigma}, \beta_{l} \mid \boldsymbol{\sigma}, \beta_{k}\right) \\
\forall \beta_{l} \neq \beta_{k} \in\left\{\beta_{1}, \ldots, \beta_{K}\right\}, \\
\mathscr{M}\left(\boldsymbol{\sigma}, \beta_{k}, \varepsilon \mid \boldsymbol{\sigma}, \beta_{k}, \varepsilon\right)=1-\sum_{l \neq k} \mathscr{M}\left(\boldsymbol{\sigma}, \beta_{l}, \varepsilon \mid \boldsymbol{\sigma}, \beta_{k}, \varepsilon\right) .
\end{gathered}
$$

The inter-replica transition probability of the TCV solution is then given by

$$
\begin{aligned}
& \Lambda\left(\boldsymbol{\sigma}, \beta_{k},-\varepsilon \mid \boldsymbol{\sigma}, \beta_{k}, \varepsilon\right)_{\mathrm{IMGS}} \\
& \quad=\max \left[0, \sum_{l \neq k}\left(\mathscr{M}\left(\boldsymbol{\sigma}, \beta_{l},-\varepsilon \mid \boldsymbol{\sigma}, \beta_{k},-\varepsilon\right)-\mathscr{M}\left(\boldsymbol{\sigma}, \beta_{l}, \varepsilon \mid \boldsymbol{\sigma}, \beta_{k}, \varepsilon\right)\right)\right] .
\end{aligned}
$$

To sample from $\pi(\beta \mid \sigma)$ with the IMGS, one follows the same steps as in Algorithm 3, except for making use of (31) and (32) in steps (5) and (6). We have demonstrated ${ }^{36}$ in the context of the 1D Potts model that the optimality of MGS over the standard Gibbs sampler is modestly replicated in their irreversible counterparts with the SDBC. It is therefore of interest to inspect if similar improvement is replicated in the context of simulated tempering. 


\section{ALGORITHM 3. Simulated tempering with IGS.}

$$
\begin{aligned}
& \text { Input: Initialize } \tilde{X}^{(0,0)}=\left(\boldsymbol{\sigma}, \beta^{(0)}, \varepsilon^{(0)}\right) \\
& \text { 1: For } t=0, \ldots, \mathscr{T}-1 \\
& \text { 2: For } \tau=0, \ldots, \Gamma-1 \\
& \text { 3: Sample from } \tilde{\pi}(\boldsymbol{\sigma} \mid \beta, \varepsilon) \text { : Perform an MC or MD simulation to } \\
& \quad \text { update } \tilde{X}^{(t, \tau)}=\left(\boldsymbol{\sigma}, \beta^{(t)}, \varepsilon^{(t)}\right) \text { to } \tilde{X}^{(t, \tau+1)}=\left(\boldsymbol{\sigma}^{\prime}, \beta^{(t)}, \varepsilon^{(t)}\right), \boldsymbol{\sigma}, \boldsymbol{\sigma}^{\prime} \in \Omega \text {. } \\
& \text { 4: end for } \\
& \text { 5: Sample from } \tilde{\pi}(\beta \mid \boldsymbol{\sigma}, \varepsilon): \text { Suppose that } \tilde{X}^{(t, \Gamma)}=\left(\boldsymbol{\sigma}, \beta_{k}, \varepsilon\right) \text {, assign } \\
& \tilde{X}^{(t+1, \Gamma)}=\left(\boldsymbol{\sigma}, \beta_{l}, \varepsilon\right), \beta_{l} \in\left\{\beta_{1}, \ldots, \beta_{K}\right\} \text {, with the transition } \\
& \text { probability } \\
& \quad \mathscr{G}\left(\boldsymbol{\sigma}, \beta_{l}, \varepsilon \mid \boldsymbol{\sigma}, \beta_{k}, \varepsilon\right)=\Theta\left(\beta_{l}, \varepsilon \mid \beta_{k}, \varepsilon\right) G\left(\boldsymbol{\sigma}, \beta_{l} \mid \boldsymbol{\sigma}, \beta_{k}\right) \quad \forall \beta_{l} \neq \beta_{k}, \\
& \qquad \mathscr{G}\left(\boldsymbol{\sigma}, \beta_{k}, \varepsilon \mid \boldsymbol{\sigma}, \beta_{k}, \varepsilon\right)=1-\sum_{l \neq k} \mathscr{G}\left(\boldsymbol{\sigma}, \beta_{l}, \varepsilon \mid \boldsymbol{\sigma}, \beta_{k}, \varepsilon\right) .
\end{aligned}
$$

6: If $\beta_{l}=\beta_{k}$, assign $\tilde{X}^{(t+1, \Gamma)}=\left(\sigma, \beta_{k},-\varepsilon\right)$ with the transition probability

$$
P\left(\boldsymbol{\sigma}, \beta_{k},-\varepsilon \mid \boldsymbol{\sigma}, \beta_{k}, \varepsilon\right)=\frac{\Lambda\left(\boldsymbol{\sigma}, \beta_{k},-\varepsilon \mid \boldsymbol{\sigma}, \beta_{k}, \varepsilon\right)_{\mathrm{IGS}}}{1-\sum_{r \neq k} \mathscr{G}\left(\boldsymbol{\sigma}, \beta_{r}, \varepsilon \mid \boldsymbol{\sigma}, \beta_{k}, \varepsilon\right)} .
$$

7: If this is also rejected, then set $\tilde{X}^{(t+1, \Gamma)}=\tilde{X}^{(t, \Gamma)}$.

8: $\tilde{X}^{(t+1, \Gamma)}=\left(\sigma, \beta^{(t+1)}, \varepsilon^{(t+1)}\right) \rightarrow \tilde{X}^{(t+1,0)}$

9: end for

\section{PERFORMANCE ANALYSIS WITH MCMC SIMULATIONS}

We carry out simulated tempering simulations to test the performance of IGS and IMGS when sampling from $\tilde{\pi}(\beta \mid \sigma, \varepsilon)$. In this section, we perform MCMC simulations to sample from $\tilde{\pi}(\sigma \mid \beta, \varepsilon)$. Performance analysis whereby MD simulations are used to sample from $\tilde{\pi}(\boldsymbol{\sigma} \mid \beta, \varepsilon)$ are provided in the section titled Performance analysis with MD simulations.

To demonstrate the validity of our algorithms, we first consider a system described by a one-dimensional double well potential whose exacts weights $w_{k}$ for $k=1, \ldots, K$ are known. We show that both the IGS and IMGS sample from the correct distribution at a given $\beta$. In the Subsection titled Ising model, we consider a more complex test system, the Ising model. For the Ising model, we demonstrate that both the IGS and IMGS improve the relaxation dynamics of inverse temperature and some system observables when compared to their respective reversible counterparts. We also show that the relaxation dynamics of inverse temperature and system observables can be significantly better than those of $\mathrm{MH}$ and IMH algorithms. In this section, we use $\beta=1 / k_{B} T$, whereby the Boltzmann constant $k_{B}$ is set to 1 .

In this paper, we define the integrated autocorrelation time $\tau_{\text {int }, f}$ for a system observable $f$ as

$$
\tau_{\text {int }, f}=1+2 \sum_{t=1}^{\infty} C_{f}(t)
$$

where $C_{f}(t)$ is the autocorrelation function given by

$$
C_{f}(t)=\frac{\mathrm{E}_{\pi}\left[f\left(t^{\prime}+t\right) f\left(t^{\prime}\right)\right]-\mathrm{E}_{\pi}\left[f\left(t^{\prime}\right)\right]^{2}}{\mathrm{E}_{\pi}\left[f^{2}\left(t^{\prime}\right)\right]-\mathrm{E}_{\pi}\left[f\left(t^{\prime}\right)\right]^{2}}
$$

and we set $t^{\prime}$ sufficiently large for equilibration to estimate $C_{f}(t)$. $\mathrm{E}_{\pi}[\cdots]$ is understood to be the expectation value with respect to the target distribution $\pi(\sigma, \beta)$. Note that the expectation value $\mathrm{E}_{\tilde{\pi}}[f]$ with respect to the extended target distribution $\tilde{\pi}(\sigma, \beta, \varepsilon)$ is equivalent to the expectation value with respect to the original distribution $\pi(\sigma, \beta)$,

$$
\begin{aligned}
\mathrm{E}_{\tilde{\pi}}[f] & =\sum_{\tilde{\Omega}} \tilde{\pi}(\boldsymbol{\sigma}, \beta, \varepsilon) f(\boldsymbol{\sigma}, \beta, \varepsilon) \\
& =\sum_{\varepsilon^{\prime}} \sum_{\bar{\Omega}} \tilde{\pi}\left(\boldsymbol{\sigma}, \beta, \varepsilon^{\prime}\right) f\left(\boldsymbol{\sigma}, \beta, \varepsilon^{\prime}\right) \\
& =\sum_{\varepsilon^{\prime}} \sum_{\bar{\Omega}} \frac{1}{2} \pi(\boldsymbol{\sigma}, \beta) f(\boldsymbol{\sigma}, \beta) \\
& =\sum_{\bar{\Omega}} \pi(\boldsymbol{\sigma}, \beta) f(\boldsymbol{\sigma}, \beta) \\
& =\mathrm{E}_{\pi}[f],
\end{aligned}
$$

where we have made use of (16) and have assumed that $f(\sigma, \beta, \varepsilon)$ $=f(\sigma, \beta,-\varepsilon)=f(\sigma, \beta)$. Consider the measurements $f_{1}, \ldots, f_{N}$ of the observable $f$. A large integrated autocorrelation time of the observable $f$ is indicative of a large corresponding asymptotic variance on the expectation value for $f$. As often, the relationship $\sigma_{f}^{2}=\sigma_{0, f}^{2} \tau_{\text {int }, f}$ is used to compute the asymptotic variance $\sigma_{f}^{2} . \sigma_{0, f}^{2}$ is the naive variance on the expectation value of $f$, that is, the variance on the expectation value of $f$ by treating all the realizations $f_{1}, \ldots, f_{N}$ as though they were independently sampled.

In simulated and parallel tempering simulations, we are often interested in computing expectation values of system observables under the distribution $\pi(\sigma \mid \beta)$ given in (1) at a single temperature of interest, often the coolest temperature. The simplest method of achieving this is to discard all measurements made at temperatures other than the temperature of interest and thereby make use of only a fraction of the data generated. However, perhaps a more cost effective method is to use one of the re-weighting techniques ${ }^{48,49}$ to properly weight the data generated at all temperatures in order to compute expectations under $\pi(\sigma \mid \beta)$ for any given temperature of interest. In other words, all configurations sampled from the joint distribution $\pi(\sigma, \beta)$ in (2) can be reweighed to compute expectations at a given temperature of interest. Within a fixed computational time, it is therefore of interest to collect as large number of uncorrelated samples as possible from the joint $\pi(\sigma, \beta)$ in order to compute (using reweighing techniques) expectation values with small variance at a given temperature of interest. The quantity $N / \tau_{\text {int }}$ is often used to establish the effective sample size, i.e., the number of uncorrelated samples in the time series data consisting of $N$ measurements. The integrated autocorrelation time therefore not only quantifies the relaxation dynamics of a given system observable but also can be used to test the sampling efficiency of a given algorithm. Among other comparison tools, we will therefore make use of the integrated autocorrelation time to provide comparison of 
the sampling efficiency of our methods to other methods currently in use.

\section{D model potential}

We consider a simple system described by the 1D double well potential

$$
U(x)=C(x+1)^{2}(x-1)^{2}, \quad x \in \Omega,
$$

with energy minima at coordinates $x=+1$ and $x=-1$ and an energy barrier of magnitude $C \geqslant 0$ at $x=0$. To sample from $\tilde{\pi}(\sigma \mid \beta, \varepsilon)$, we perform a Metropolis-Hastings MC simulation at fixed $\beta$ using a Gaussian proposal in the $x$-coordinate,

$$
x_{i+1}^{\prime}=x_{i}+\varsigma \xi
$$

to propose $x^{\prime} \in \Omega$ for the $(i+1)$ th Monte-Carlo time step. $\xi \sim \mathscr{N}(0,1)$ and the standard deviation $\varsigma$ is fixed at 0.05 . To sample from $\tilde{\pi}(\beta \mid \sigma, \varepsilon)$, we use a predetermined set of $K$ inverse temperatures that are equally spaced in the range $\beta_{1}=1$ and $\beta_{K}=0.1$. The weights are then numerically computed using

$$
w_{k}=-\ln Z\left(\beta_{k}\right)=-\ln \int_{-\infty}^{+\infty} d x e^{-\beta_{k} U(x)}
$$

Simulated tempering is then performed by alternately sampling from the conditional distributions $\tilde{\pi}(\sigma \mid \beta, \varepsilon)$ and $\tilde{\pi}(\beta \mid \sigma, \varepsilon)$ such that a single step $t$ involves $\Gamma$ Monte-Carlo steps to sample from $\tilde{\pi}(\sigma \mid \beta, \varepsilon)$ and a single MC step to sample from $\tilde{\pi}(\beta \mid \boldsymbol{\sigma}, \varepsilon)$.

We have performed two sets of simulated tempering simulations with $K=30$ and $K=512$ temperature domain sizes. The results are shown in Figs. 1 and 2, respectively. For comparison, we show the performance of IMGS against the standard Metropolis-Hastings algorithm as given in Algorithm 2 and the irreversible MetropolisHastings (IMH) as proposed by Sakai and Hukushima. ${ }^{30}$ For both $\mathrm{MH}$ and the IMH algorithms, we had adopted the nearest neighbor exchange scheme for $\beta$. The tunable deviation parameter $\delta$ is set to 1 for both IMH and IMGS.

The top row of Fig. 1 shows the evolution of $\beta$ and the position coordinate $x$ as a function of the MC steps $t$. Expectedly, the trajectory of $\beta$ for $\mathrm{MH}$ indicates that the system performs a characteristically random walk in the temperature space. The IMH algorithm, on the other hand, was proposed to suppress diffusive behavior in the temperature space, and we therefore observe the typically deterministic exploration of $\beta$ coordinates with a visually better mixing rate than MH. The IMGS seems to provide a more ballistic exploration of temperature space, as shown in Fig. 1(c). Unlike MH and $\mathrm{IMH}$, which perform optimally with nearest neighbor $\beta$ proposals, the IMGS can perform more distant jumps in the $\beta$ coordinate, thus inducing a more global exploration of temperature space.

From the autocorrelation functions $C_{\beta}(t)$ for $\beta$, shown in Fig. $1(\mathrm{~g})$, we observe that $C_{\beta}(t)$ for IMGS decays more rapidly compared to $\mathrm{MH}$ and IMH. In particular, we find that $\left[\tau_{\text {int }, \beta}\right]_{\mathrm{MH}} /\left[\tau_{\text {int }, \beta}\right]_{\mathrm{IMGS}} \sim 87.6$ and $\left[\tau_{\text {int }, \beta}\right]_{\mathrm{IMH}} /\left[\tau_{\text {int }, \beta}\right]_{\mathrm{IMGS}} \sim 5.2$. Generally, an improvement in the relaxation dynamics of $\beta$ is accompanied by an improvement in the relaxation dynamics of system observables. From the trajectory of position coordinate $x$, we clearly observe that an improvement in the mixing rate of $\beta$ induces a more frequent crossing of the energy barrier at $x=0$ (Fig. 1, top row). The energy and position coordinate autocorrelation functions, $C_{\mathscr{E}}(t)$ [Fig. 1(h)] and $C_{x}(t)$ [Fig. 1(i)], respectively, therefore decay most rapidly for the IMGS. Note that $C_{x}(t)$ for the IMGS decays substantially faster than that for $\mathrm{MH}$, but compared to that for $\mathrm{IMH}$, there is little gain. This is in contrast to the autocorrelation function $C_{\mathscr{E}}(t)$ for energy, which decays most rapidly for the IMGS with a distinctly clear gain over that of IMH. We speculate that perhaps for a given domain size $K$, there could possibly be an optimum mixing rate for $\beta$ such that any further improvement in the mixing rate for $\beta$ may not necessarily lead to substantial improvement in the mixing rate for $x$.

In Fig. 2, we consider simulations for a larger temperature domain, $K=512$. The improvement in performance of IMGS compared to $\mathrm{MH}$ and $\mathrm{IMH}$ is now more visibly clear. The trajectory of $\beta$ for $\mathrm{MH}$ [Fig. 2(a)] confirms the expected degradation in exploration of temperature space, which is typical of random walks on domains of increasing size. For the IMH algorithm, the deterministic exploration of temperature space in a specific direction [Fig. 2(b)] is now visibly clearer than that for the $K=30$ case [Fig. 1(b)]. Notice that this behavior leads to a very uniform exploration of $\beta$ coordinates, as shown in the probability distribution of inverse temperature [Fig. 2(e)]. In contrast, the IMGS mixing rate for $\beta$ remains visibly unchanged with increasing temperature domain. In fact, notice that the autocorrelation functions $C_{\beta}(t), C_{\mathscr{E}}(t)$, and $C_{x}(t)$ (Fig. 2, third row) for the IMGS are visibly unchanged from the $K=30$ case (Fig. 1, third row). This observation is in agreement with a similar study by Chodera and Shirts ${ }^{38}$ who show numerically that the integrated autocorrelation time for temperature and position index remain independent of the temperature domain size $K$ for algorithms based on Gibbs sampling. In particular, we report $\left[\tau_{\text {int }, \beta}\right]_{\mathrm{MH}} /\left[\tau_{\text {int }, \beta}\right]_{\mathrm{IMGS}} \sim 1.25 \times 10^{4}$ and $\left[\tau_{\text {int }, \beta}\right]_{\mathrm{IMH}} /\left[\tau_{\text {int }, \beta}\right]_{\mathrm{IMGS}} \sim 79.2$. Consequently, the speedup in $\tau_{\text {int }, x}$ is $\left[\tau_{\text {int }, x}\right]_{\mathrm{MH}} /\left[\tau_{\text {int }, x}\right]_{\mathrm{IMGS}} \sim 14.7$ and $\left[\tau_{\text {int }, x}\right]_{\mathrm{IMH}} /\left[\tau_{\text {int }, x}\right]_{\mathrm{IMGS}} \sim 3.4$. In fact, for the MH algorithm, the relaxation dynamics of $\beta$ is diffusive, and therefore, $\tau_{\text {int }, \beta}$ scales on the order of $\mathscr{O}\left(K^{2}\right)$. For the IMH algorithm, it has been demonstrated $^{30}$ that the relaxation dynamics of $\beta$ scales on the order of $\mathscr{O}(K)$, i.e., a square root reduction in the dynamical scaling of relaxation time with respect to the temperature domain size. In the section titled Ising model, we have shown using the Ising model as a test bed that for the IMGS, the relaxation dynamics of $\beta$ and system observables is independent of $K$ and scales on the order of $\mathscr{O}(1)$.

Furthermore, we demonstrate in the bottom row of Figs. 1 and 2 that the IMGS leaves the target distribution invariant. We have shown convergence to the correct free energy profile $\beta F(x)$ $=-\ln (\pi(x \mid \beta))$ for the temperature of interest, the coldest temperature corresponding to $\beta_{1}=1$. The performance of the IGS (not shown for this model) is very similar to IMGS. The gain in integrated autocorrelation times for IMGS is only slightly better than those of IGS. Furthermore, for this simple model, we have not shown comparison of IMGS to that of its reversible counterpart, the MGS. The gain in integrated autocorrelation time of $\beta, \mathscr{E}$, and $x$ for the IMGS is only slightly better than that of MGS.

We have demonstrated the validity of our method using this simple $1 \mathrm{D}$ potential model and have provided first hand comparison with the widely used $\mathrm{MH}$ method and the recently proposed IMH 

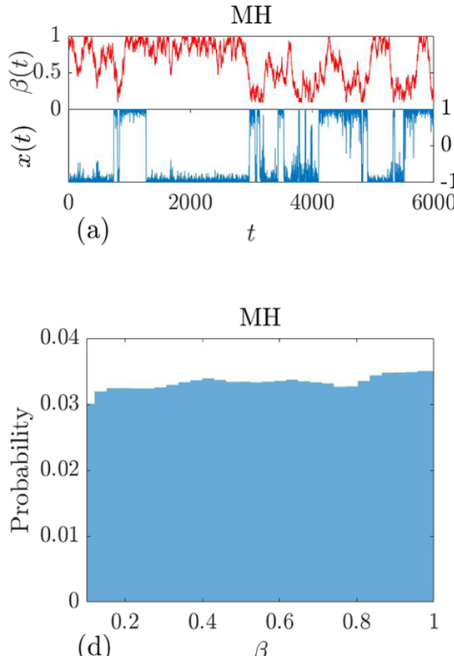

(d)

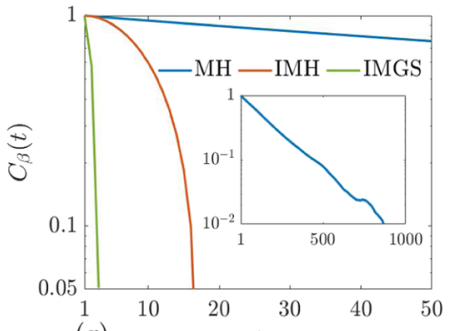

(g)

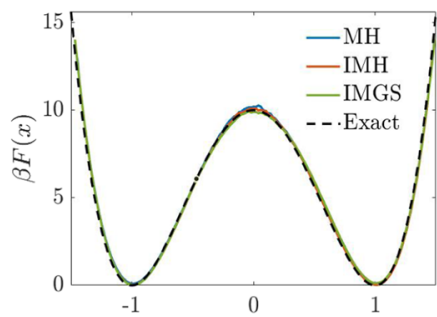

(j)
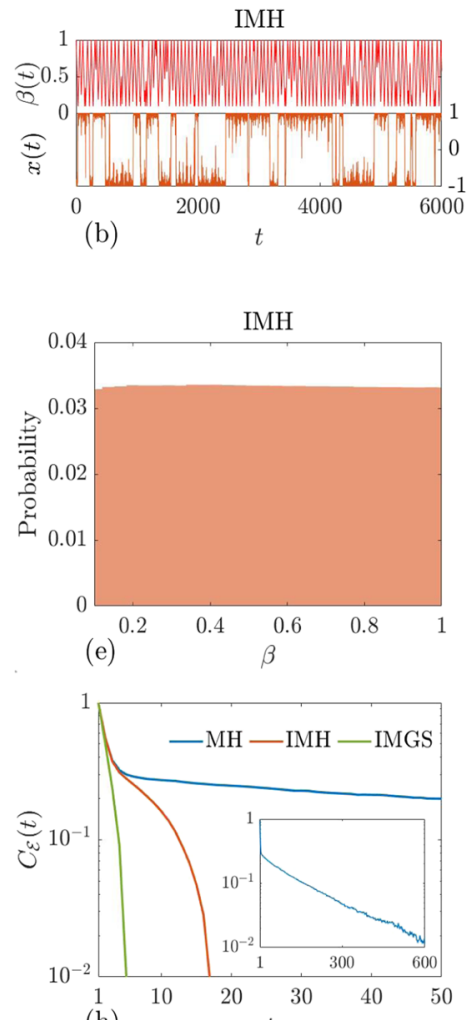

(h)

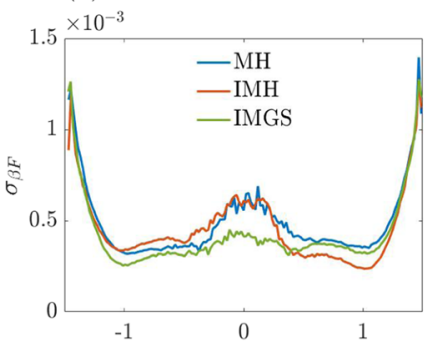

(k)
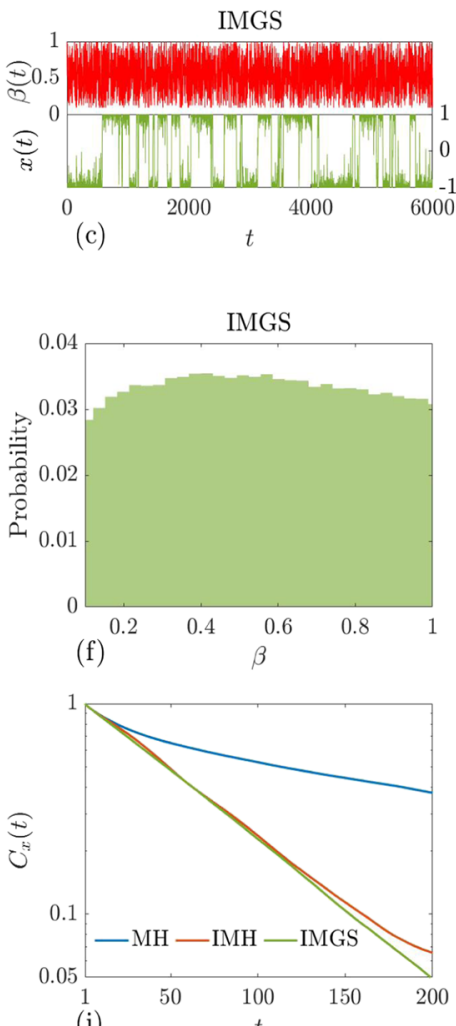

(i)

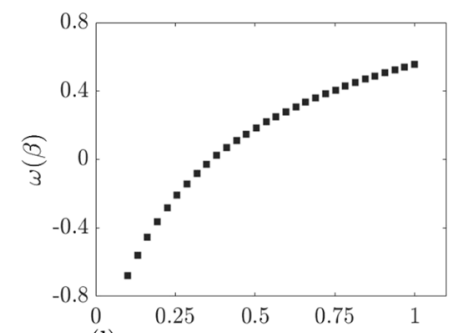

(1)

FIG. 1. Simulations of a simple system described by the 1D model potential given in (38) for the temperature domain size $K=30$. [(a)-(c)] Trajectories of inverse temperature $\beta$ and position coordinate $x$ as a function of MC step $t$. [(d)-(f)] Probability of inverse temperatures obtained from frequency distributions. [(g)-(i)] Autocorrelation functions $C(t)$ for inverse temperature $\beta$, energy $\mathscr{E}(x)$, and position coordinate $x$. [(j)-(l)] Free energy profile $\beta F(x)=-\ln (\pi(x \mid \beta))$ at the coldest temperature $\beta=1$ (left), standard error on the free energy profile trajectories obtained from 100 independent simulations (center), and the weights $\omega(\beta)$ computed numerically using (40) (right). The simulation parameters are $\Gamma=10^{2} \mathrm{MC}$ steps, $\mathscr{T}=10^{6} \mathrm{MC}$ steps, $\beta_{1}=1$ and $\beta_{K}=0.1$ all equally spaced, and energy barrier height $C=10$ in $(38)$. The simulations were initialized with $\beta^{(0)}=1$ and $x^{(0)}=-1$ and a random assignment of $\varepsilon \in\{-1,+1\}$. The deviation parameter $\delta$ is set to 1 for both the IMH and IMGS.

algorithm of Sakai and Hukushima. ${ }^{30}$ The potential gains of IMGS over its reversible counterpart are, however, not fully captured by this simple model. In the section titled Ising model, we observe that for a more complex and practical system, we observe a clear gain of IMGS over its reversible counterpart.

\section{Ising model}

In this section, we test our methods on a $L \times L 2 \mathrm{D}$ Ising model ${ }^{4}$ with periodic boundary conditions. The Hamiltonian $H(\boldsymbol{\sigma})$ of the
Ising model is given by

$$
H(\boldsymbol{\sigma})=-\sum_{\langle i, j\rangle} J_{i j} \sigma_{i} \sigma_{j},
$$

where the notation $\langle i, j\rangle$ indicates that spins $\sigma_{i}, \sigma_{j} \in\{+1,-1\}$ are nearest neighbors. We set the interaction strength to constant so that $J_{i j}=J=1$. A given configuration or the state of the model is defined by the state vector $\boldsymbol{\sigma}=\left(\sigma_{1}, \ldots, \sigma_{N}\right) \in \Omega$ with $N=L^{2}$ spins. The discrete state space $\Omega=\{1, \ldots, S\}$ therefore consists of $S=2^{N}$ configurations. We consider a 2D lattice of row index $m$ and column index 

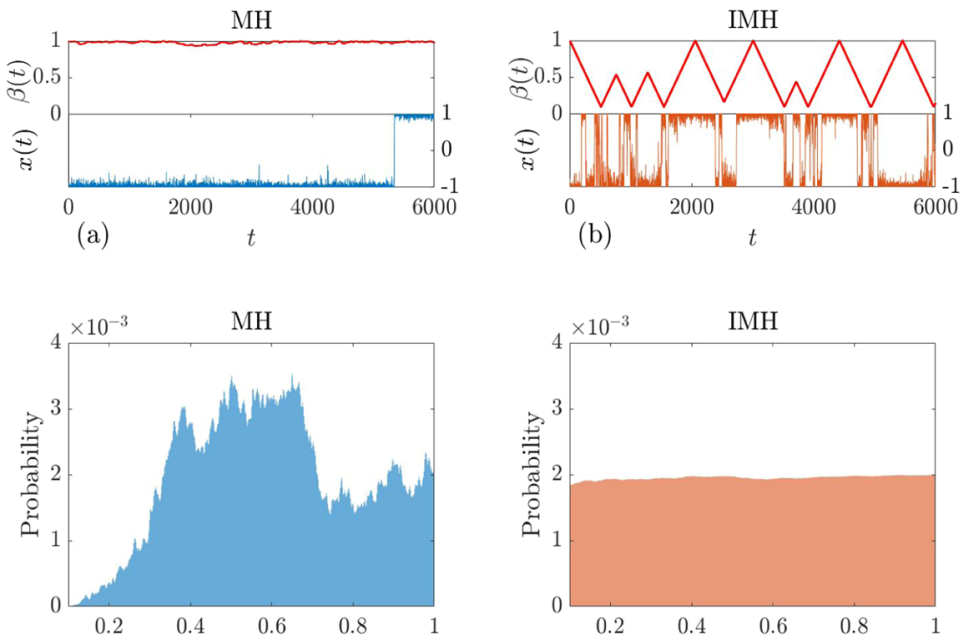

(d)

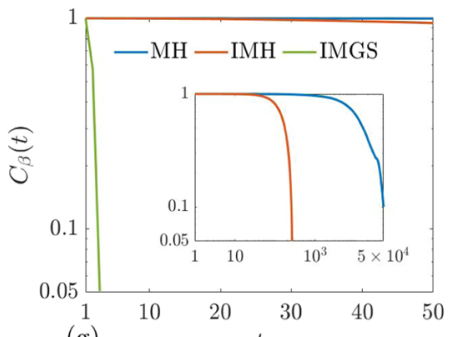

(g)

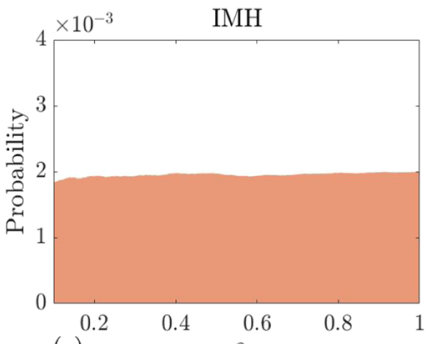

(e)

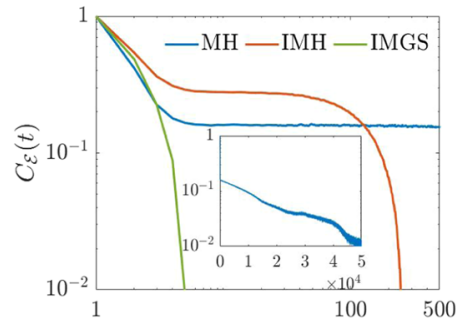

(h)

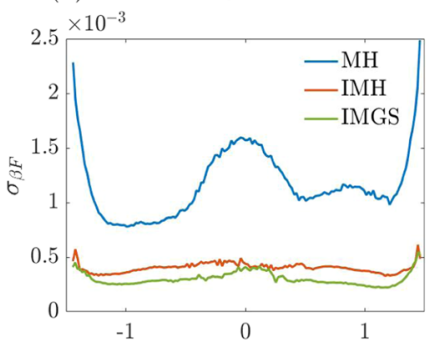

$(\mathrm{k})$
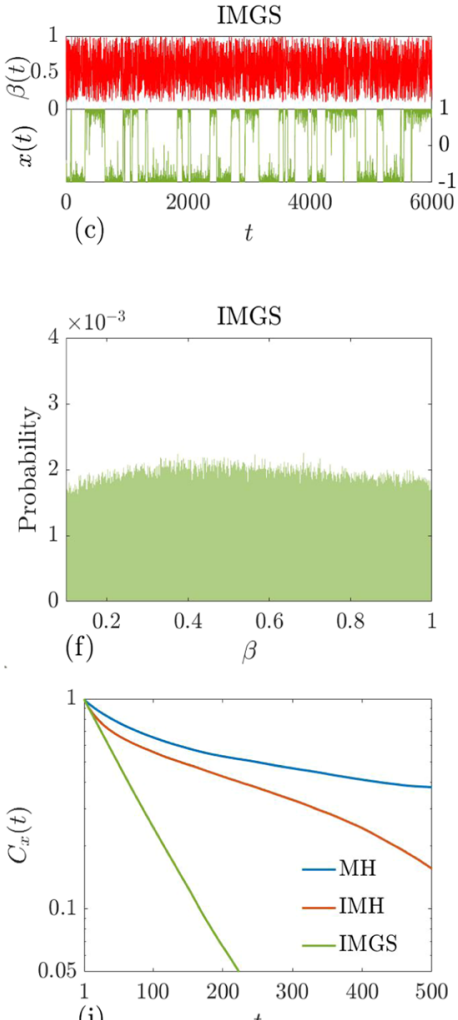

(i)

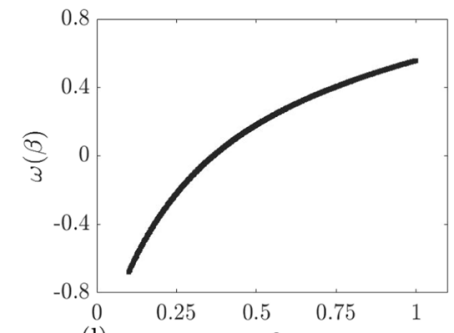

(1)

FIG. 2. Simulations of a simple system described by the $1 \mathrm{D}$ model potential given in (38) for temperature domain size $K=512$. [(a)-(c)] Trajectories of inverse temperature $\beta$ and position coordinate $x$ as a function of MC step $t$. [(d)-(f)] Probability of inverse temperatures obtained from frequency distributions. [(g)-(i)] Autocorrelation functions $C(t)$ for inverse temperature $\beta$, energy $\mathscr{E}(x)$, and position coordinate $x$. [(j)-(l)] Free energy profile $\beta F(x)=-\ln (\pi(x \mid \beta))$ at the coldest temperature $\beta=1$ (left), standard error on the free energy profile trajectories obtained from 100 independent simulations (center), and the weights $\omega(\beta)$ computed numerically using (40) (right). The simulation parameters are $\Gamma=10^{2} \mathrm{MC}$ steps, $\mathscr{T}=10^{6} \mathrm{MC}$ steps, $\beta_{1}=1$ and $\beta_{K}=0.1$ all equally spaced, and energy barrier height $C=10 \mathrm{in}(38)$. The simulations are initialized with $\beta^{(0)}=1$ and $x^{(0)}=-1$ and a random assignment of $\varepsilon \in\{-1,+1\}$. The deviation parameter $\delta$ is set to 1 for both IMH and IMGS.

$n$, and the energy density $\mathscr{E}$ of the system is then defined by

$$
\mathscr{E}=-J / N \sum_{m, n}\left(\sigma_{m, n} \sigma_{m+1, n}+\sigma_{m, n} \sigma_{m, n+1}\right),
$$

where periodic boundary conditions are imposed so that $\sigma_{m, L+1}$ $=\sigma_{m, 1}$ and $\sigma_{L+1, n}=\sigma_{1, n}$. The magnetization density of the system is defined as $m=1 / N \sum_{i} \sigma_{i}$, and the magnetic susceptibility $\chi$ is given by

$$
\chi=\frac{1}{N}\left\|\sum_{i} \sigma_{i}\right\|^{2}
$$

We perform simulated tempering by alternately sampling from the distributions $\tilde{\pi}(\boldsymbol{\sigma} \mid \beta, \varepsilon)$ and $\tilde{\pi}(\beta \mid \boldsymbol{\sigma}, \varepsilon)$. We define one sweep of the $2 \mathrm{D}$ spin lattice as $N$ MC trials to update individual spins. To update individual spins, we make use of the Metropolis Monte Carlo algorithm $^{4}$ with the sequential updating scheme, ${ }^{50}$ whereby individual spins are updated in a fixed sequential order. $\Gamma$ sweeps are performed at fixed $\beta$ to sample from $\tilde{\pi}(\boldsymbol{\sigma} \mid \beta, \varepsilon)$, taking measurements at each sweep, before attempting a single Monte Carlo step to sample from $\tilde{\pi}(\beta \mid \boldsymbol{\sigma}, \varepsilon)$. The weights $\omega(\beta)$ are determined using the method proposed by Park and Pande. 
We have verified the correctness of our irreversible simulated tempering algorithms IGS and IMGS and have obtained near perfect agreement for energy density $\mathscr{E}$, magnetization density $m$, and specific heat capacity $c$ (Fig. 3, top row) with the Metropolized-Gibbs sampler. ${ }^{35,38}$ As an additional baseline comparison, we have also computed the corresponding values of the three observables using a much longer standard Metropolis-Hastings MC simulation with no simulated tempering (shown in red crosses). Notice that the critical inverse temperature $\beta_{c} \simeq 0.4407$ of the model is within our predetermined set of temperatures for the simulation: $\beta_{1}=0.5$ to $\beta_{K}=0.2$, all equally spaced. The deviation parameter $\delta$ in the skewness function given in (28) determines the extent to which $\mathrm{DBC}$ is violated, and $\delta=0$ recovers DBC. With $\delta=0$, the IGS, as given in Algorithm 3, decomposes to its reversible counterpart, the standard Gibbs sampler with transition probability given in (11). Likewise, the IMGS with transition in (31) breaks down to its reversible counterpart with

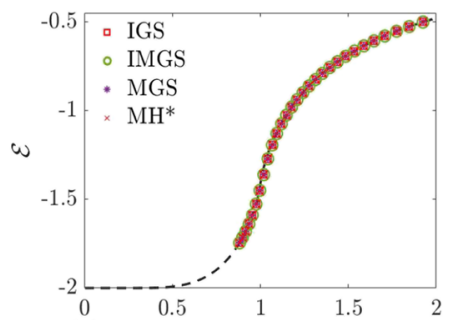

(a)
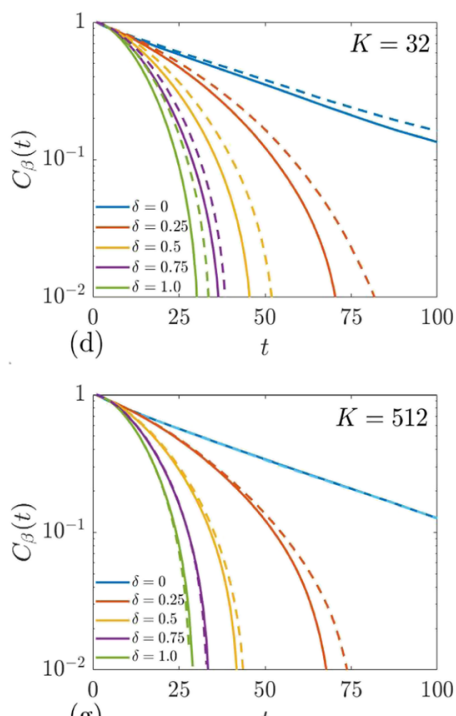

(g)

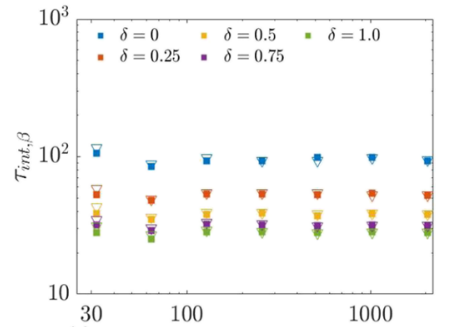

(j)
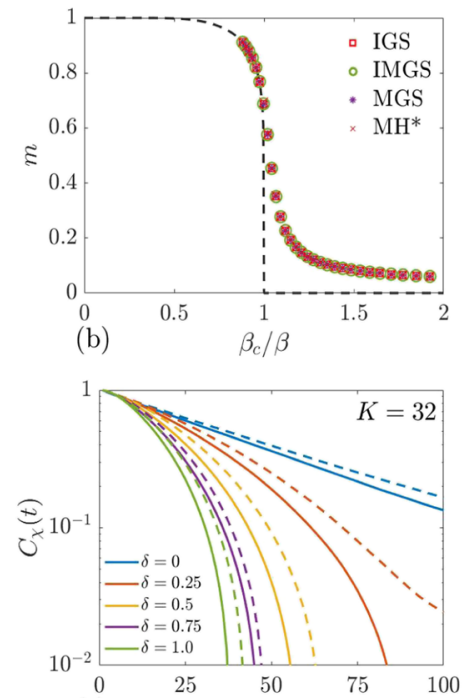

(e)

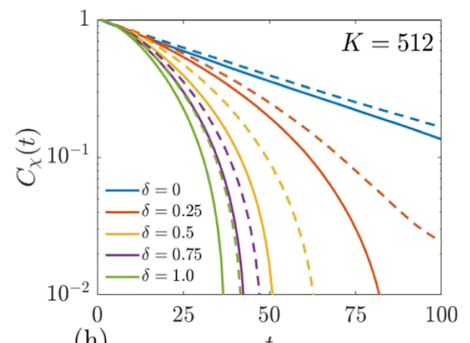

(h)

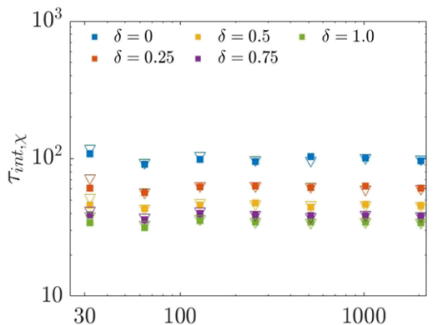

$(\mathrm{k})$
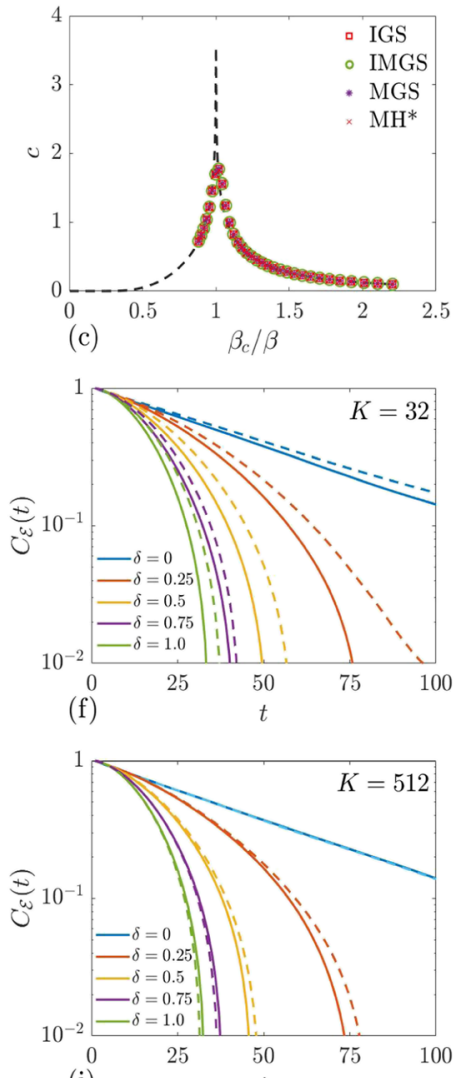

(i)

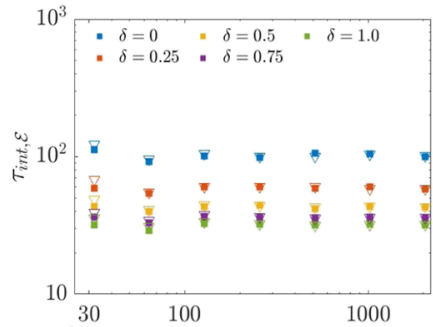

(1)

FIG. 3. Simulation results for the $25 \times 25$ Ising model with periodic boundary conditions. The simulation parameters are $\Gamma=10^{3}$ sweeps and $\mathscr{T}=10^{6} \mathrm{MC}$ steps. $\beta_{1}=0.5$ and $\beta_{K}=0.2$, all equally spaced. [(a)-(c)] Energy density $\mathscr{E}$, magnetization density $m$, and specific heat capacity $c$ obtained from simulated tempering simulations. The values obtained with our methods IGS (red squares) and IMGS (green circles) are in perfect agreement with the well-established MGS (purple stars). Also shown (red crosses) are the values obtained from a much longer Metropolis-Hastings MC simulation with no simulated tempering, which we have denoted as MH* to distinguish from results with simulated tempering. The dashed lines are Onsager's ${ }^{51}$ exact solutions for a 2D lattice of infinite dimensions. [(d)-(i)] Autocorrelation functions of $\beta, \mathscr{E}$, and $\chi$ for $K=32$ (second row) and $K=512$ (third row) for various deviation parameters $\delta$. IMGS (solid lines) and IGS (dashed lines). [(j)-(I)] Integrated autocorrelation times $\tau_{\text {int, } \beta,}, \tau_{\text {int }, \chi}$ and $\tau_{\text {int }, \mathscr{E}}$ with respect to temperature domain size $K$. IMGS (squares) and IGS (inverted triangles). 
the DBC: the Metropolized-Gibbs sampler with reversible transition probability in (15). In Fig. 3 (second and third row), we show the autocorrelation functions for $\beta, \mathscr{E}$, and $\chi$ in two temperature domain sizes, $K=32$ and $K=512$, for both of our methods IGS (dashed lines) and IMGS (solid lines). From the autocorrelation functions, we observe that in both methods, deviation from the DBC $(\delta=0)$ accelerates the relaxation dynamics of all three variables. Furthermore, the IMGS seems to provide a visibly modest improvement over the IGS for all deviation parameters. Since the implementation of IMGS comes with no additional computational cost, it is therefore recommended to choose IMGS over IGS.

To quantify the relaxation dynamics of $\beta, \mathscr{E}$, and $\chi$, we have computed the integrated autocorrelation times $\tau_{\text {int }, \beta}, \tau_{\text {int }, \chi}, \tau_{\text {int }, \mathscr{E}}$. We show these in Fig. 3 (bottom row) for various temperature domain sizes $K$ for both IGS (inverted triangles) and IMGS (squares). The numerical gain in relaxation dynamics over their reversible counterparts $(\delta=0)$ is clear for both the IGS and IMGS. In particular, we observe that $\left[\tau_{\text {int }}\right]_{\delta=0} /\left[\tau_{\text {int }}\right]_{\delta=1} \sim 3.3$ for all three variables $\beta, \mathscr{E}$, and
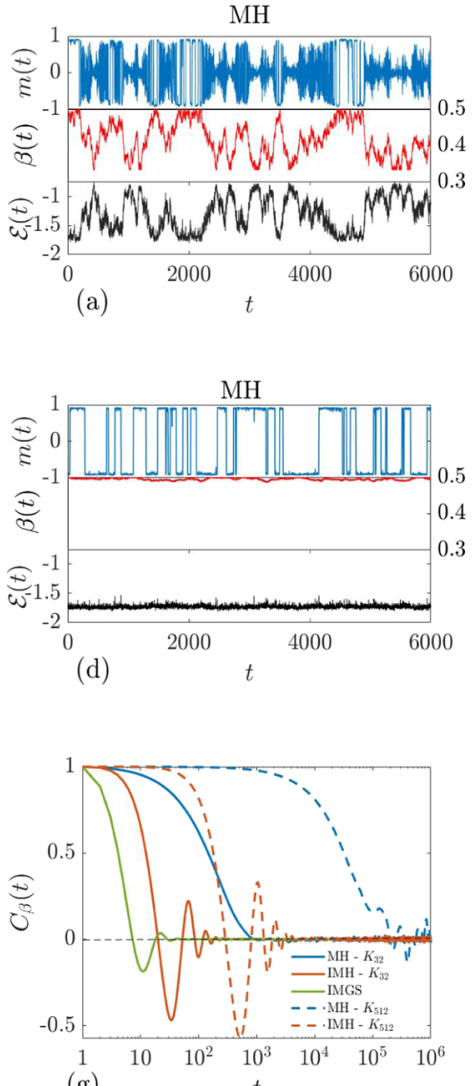

(g)

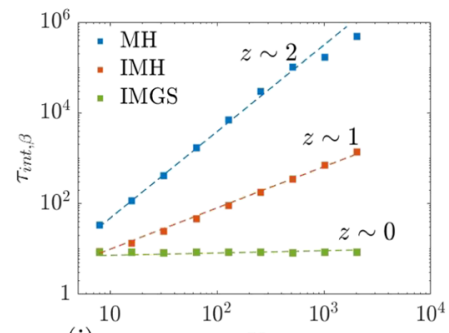

(j)
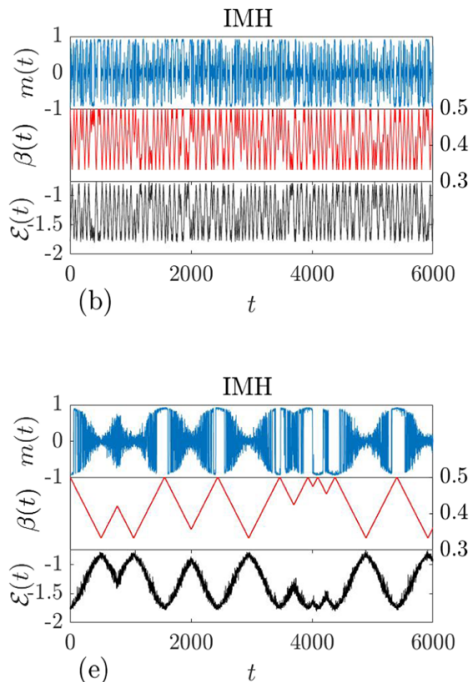

(e)

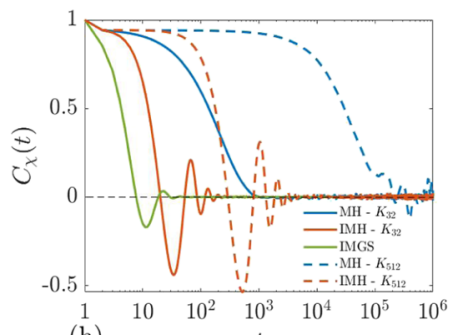

(h)

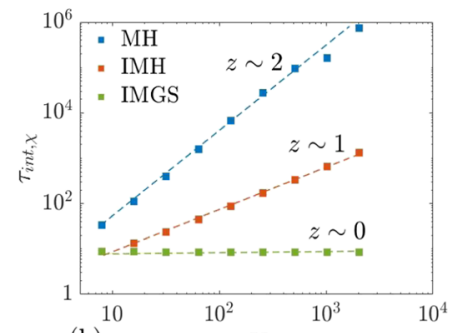

(k)

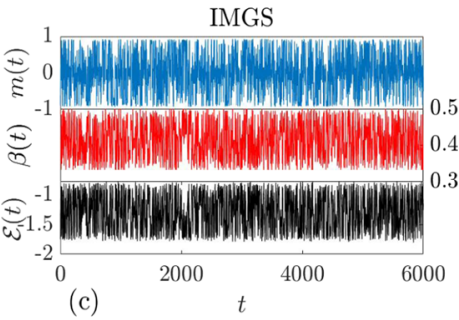

IMGS
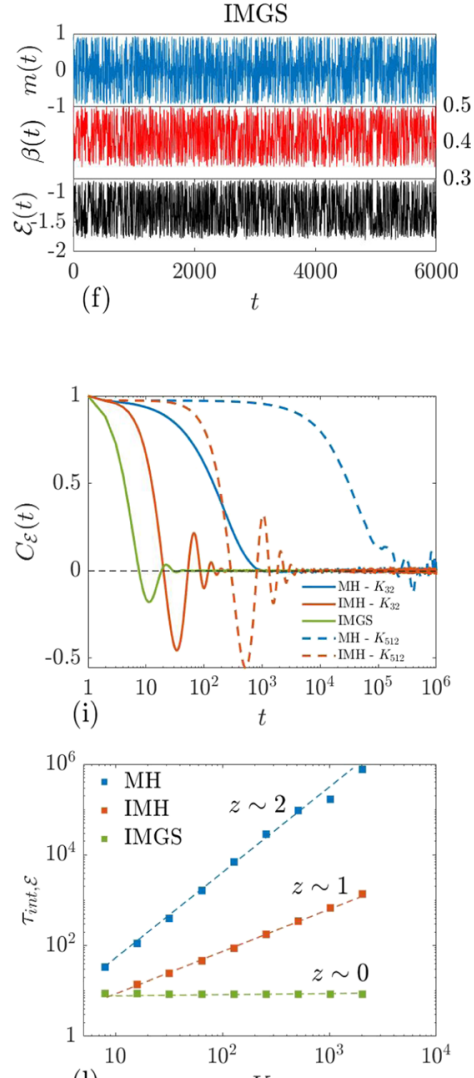

(1)

FIG. 4. Simulations results for the $12 \times 12$ Ising model with periodic boundary conditions. The simulation parameters are $\Gamma=10^{2}$ sweeps and $\mathscr{T}=10^{7}$ MC steps. $\beta_{1}=$ 0.5 and $\beta_{K}=0.33$, all equally spaced. The deviation parameter $\delta=1$ for both IMH and IMGS. [(a)-(f)] Trajectories of inverse temperature $\beta$, magnetization $m$, and energy density $\mathscr{E}$ shown for the first $6000 \mathrm{MC}$ steps $t$ for $K=32$ (top row) and $K=512$ (second row) temperatures. [(g)-(i)] Autocorrelation functions $C_{\beta}(t), C_{\chi}(t)$, and $C_{\mathscr{E}}(t)$ for the temperature domain size $K=32$ (solid lines) and $K=512$ (dashed lines). The autocorrelation functions of IMGS for the two domain sizes heavily overlap. [(j)-(I)] Dynamical scaling of the integrated autocorrelation times for $\beta, \chi$, and $\mathscr{E}$ with respect to temperature domain size $K$. The dynamical scaling exponents are retrieved using the asymptotic relationship $\tau_{\text {int }} \sim K^{z}$. The dashed lines are the least squares fit to the data. 
$\chi$ for both the IGS and IMGS. Reportedly, for all values of $\delta$, the integrated autocorrelation times remain fairly independent of the temperature domain size. This observation is consistent with a similar study using the Gibbs sampler. ${ }^{38}$ The apparent independence of $\tau_{\text {int }}$ with respect to $K$ is in contrast to both $\mathrm{MH}$ and IMH whose sampling efficiency degrades with temperature domains of increasing size $K$, as shown in Fig. 4 (bottom row).

Since the IMGS seems to be the best of our two methods, we will henceforth provide performance analysis only with the IMGS. In Fig. 4 (bottom row), we compare the integrated autocorrelation times of IMGS with those obtained from simulated tempering with the standard MH of Algorithm 2 and the IMH algorithm of Sakai and Hukushima. ${ }^{30}$ The scaling of $\tau_{\text {int }, \beta}$ with respect to the temperature domain size $K$ reveals the expected diffusive relaxation dynamics of $\beta$ for the $\mathrm{MH}$ algorithm, whereby $\tau_{\text {int, } \beta}$ scales on the order of $\mathscr{O}\left(K^{2}\right)$. It was numerically demonstrated ${ }^{30}$ that the IMH algorithm, which breaks detailed balance, provides a square root reduction in the mixing time for $\beta$, and we have reproduced this result to confirm that the relaxation dynamics of $\tau_{\text {int }, \beta}$ scales on the order of $\mathscr{O}(K)$ for IMH. The scaling of $\tau_{\text {int, } \beta}$ with respect to $K$ may be asymptotically modeled with $\tau_{\text {int }} \sim K^{z}$, the case in which we observe that the dynamical scaling exponent $z$ of the IMGS is effectively $\sim 0$ compared to $z \sim 2$ of MH and $z \sim 1$ of IMH. In other words, for the IMGS, $\tau_{\text {int }, \beta}$ scales on the order of $\mathscr{O}(1)$. A similar dynamical scaling behavior is observed for $\chi$ and $\mathscr{E}$ (Fig. 4, bottom row). Compared to the conventionally used $\mathrm{MH}$ algorithm, the IMGS provides a decisive gain in the relaxation dynamics of all three variables for all values of $K$; note that the gain accelerates with increasing $K$. Likewise, compared to the IMH algorithm, we clearly observe a decisive gain in relaxation dynamics with increasing $K$. Even for practically small $K$ values, the integrated autocorrelation times are shortened, but by a modest factor, compared to IMH. For example, the autocorrelation functions for $K=32$ (Fig. 4, third row) show that $C(t)$ decays faster compared to both $\mathrm{MH}$ and $\mathrm{IMH}$ for all three variables $\beta, \mathscr{E}$, and $\chi$.

In Fig. 4 , the trajectories of $\beta, m$, and $\mathscr{E}$ for $K=32$ (top row) and $K=512$ (second row) show a similar pattern to those in Figs. 1 and 2, respectively. While the sampling efficiency of both $\mathrm{MH}$ and IMH degrades with increasing values of $K$, that of IMGS seems to be independent of $K$. The IMGS provides a numerical gain in relaxation dynamics of $\beta$, $\chi$, and $\mathscr{E}$ compared to its reversible counterpart, the Metropolized-Gibbs sampler. Furthermore, unlike $\mathrm{MH}$ and $\mathrm{IMH}$, it seems insensitive to the increasing temperature domain size.
The implementation of IMGS in simulated tempering simulations may therefore be of interest, particularly so in simulations that may require a large temperature domain size $K$.

\section{PERFORMANCE ANALYSIS WITH MD SIMULATIONS}

In this section, we test our methods with MD simulations of alanine pentapeptide (ALA5) in explicit water. Under the assumption of the previous section that the IMGS appears to be slightly better than IGS, we will therefore provide comparison analysis of IMGS with some conventionally used simulated tempering algorithms. In this section, we set $\beta=1 / k_{B} T$, where the Boltzmann constant is given by $k_{B} \simeq 1.38 \times 10^{-23} \mathrm{~J} \mathrm{~K}^{-1}$. The weights $\omega(\beta)$ are determined using the method proposed by Park and Pande.

\section{Setup}

We constructed a simple linear model of ALA5 whereby the peptide was capped with NTER at the $\mathrm{N}$ terminus and CTER at the $\mathrm{C}$ terminus. We have performed MD simulations of our ALA5 model (Fig. 5) with the CHARMM36 force field ${ }^{66}$ in explicit water using CHARMM-GUI ${ }^{64}$ to set up the system. The system was solvated in a rectangular truncated box size of $10 \AA$ edge distance where we have used 3582 TIP3 water molecules and had added $3 \mathrm{~K}^{+}$and $3 \mathrm{Cl}^{-}$counterions to account for a $0.15 \mathrm{M} \mathrm{KCl}$ concentration. The simulations were run using NAMD $^{65}$ with a time step of 2 fs using a Langevin thermostat with a damping coefficient of $1 / p s$. We used the particle mesh Ewald method ${ }^{67}$ in the periodic boundary conditions with standard cut-off values given in the CHARMM-GUI ${ }^{64}$ protocols. We used the standard protocol for the equilibration step of CHARMM-GUI before performing any production run.

Simulated tempering was performed to alternately sample from the distributions $\tilde{\pi}(\boldsymbol{\sigma} \mid \beta, \varepsilon)$ and $\tilde{\pi}(\beta \mid \boldsymbol{\sigma}, \varepsilon)$. The distribution $\tilde{\pi}(\boldsymbol{\sigma} \mid \beta, \varepsilon)$ was sampled at a fixed temperature for $\Gamma=0.6$ ps before attempting a single MC trial to sample from $\tilde{\pi}(\beta \mid \sigma, \varepsilon)$, i.e., a single trial to update the temperature. Simulated tempering trajectories for $K=32$ and $K=512$ temperatures equally spaced between 300 and $500 \mathrm{~K}$ were therefore run for $\mathscr{T}=2 \times 10^{5}$ temperature swap attempts. This consisted of $\Gamma=0.6$ ps of MD sampling per temperature swap attempt, therefore totaling $120 \mathrm{~ns}$ of MD sampling per Markov chain. For both $K=32$ and $K=512$ temperatures, we had performed 6 independent runs with the same starting structure.

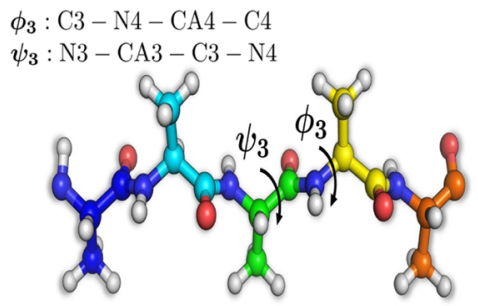

(a)

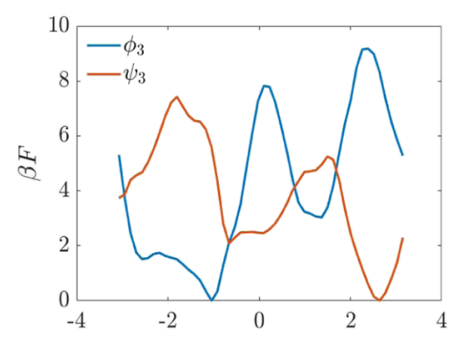

(b)

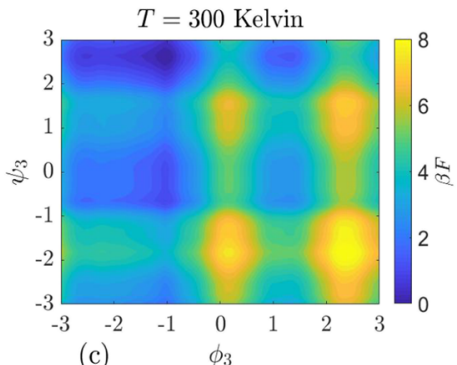

(c)

FIG. 5. (a) Alanine pentapeptide with the slowest relaxing dihedral angles $\phi_{3}$ : C3-N4-CA4-C4 and $\psi_{3}$ : N3-CA3-C3-N4, (b) free energy profiles $\beta F=-\ln (\pi(\cdot \mid \beta))$ in $\phi_{3}$ and $\psi_{3}$ obtained from a very long free MD simulation at $300 \mathrm{~K}$, and (c) $2 \mathrm{D}$ free energy landscape in $\phi_{3}$ and $\psi_{3}$ for ALA5. Units of free energy are in $\mathrm{kcal} / \mathrm{mol}$. 
Dihedral angles used as descriptors of the system were recorded at every step (2 fs resolution) of the MD simulations. For comparison, we have constructed a baseline free energy profile in the slowest relaxing dihedral angle $\phi_{3}$ : C3-N4-CA4-C4, whereby a single $2 \mu$ s long simulation at the coldest temperature was run with free MD simulation (no simulated tempering) to sample enough for every possible configuration of ALA5 [Fig. 5(b)]. The total set of dihedral angles of the system are as follows: $\phi_{1}$ : C1-N2-CA2-C2, $\phi_{2}: \mathrm{C} 2-\mathrm{N} 3-\mathrm{CA} 3-\mathrm{C} 3, \phi_{3}: \mathrm{C} 3-\mathrm{N} 4-\mathrm{CA} 4-\mathrm{C} 4$, and $\phi_{4}$ : C4-N5-CA5C5 and $\psi_{1}$ : N1-CA1-C1-N2, $\psi_{2}$ : N2-CA2-C2-N3, $\psi_{3}$ : N3-CA3C3-N4, and $\psi_{4}$ : N4-CA4-C4-N5.

\section{Results}

In Figs. 6 and 7, we show the simulation results for ALA5 for respective $K=32$ and $K=512$ temperature domain sizes. For
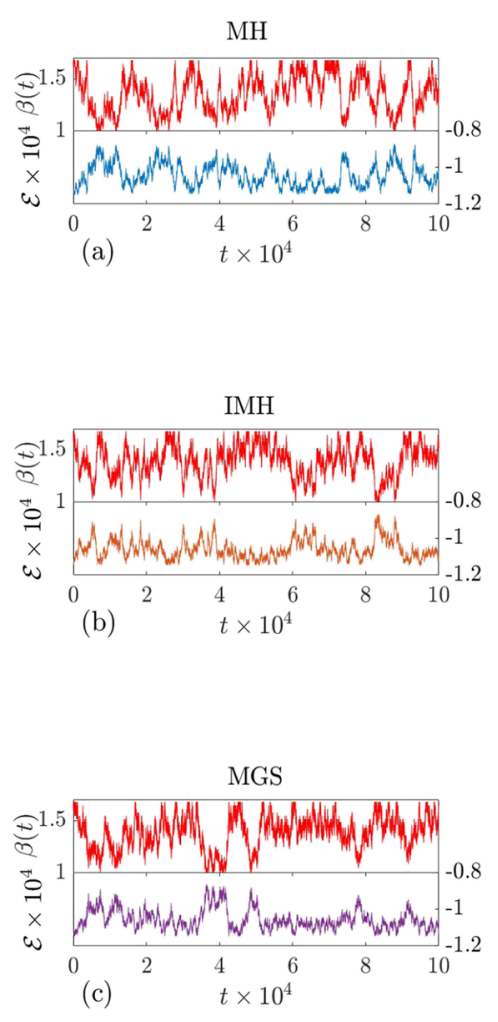

IMGS

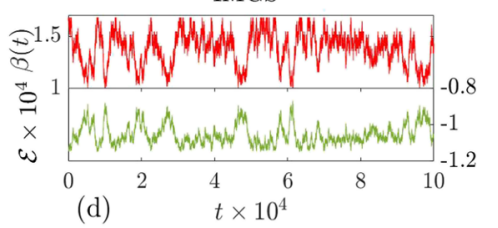

(d)

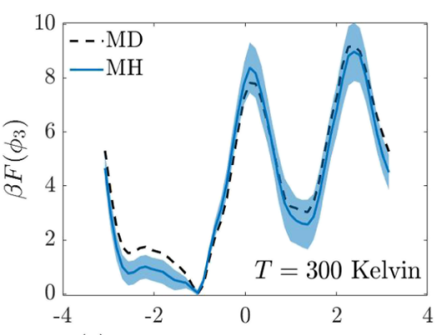

(e)

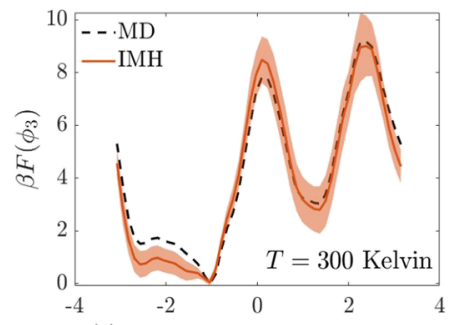

(f)

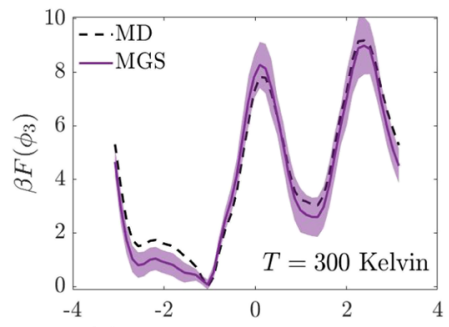

(g)

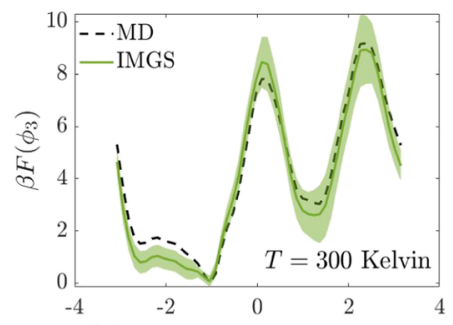

(h)

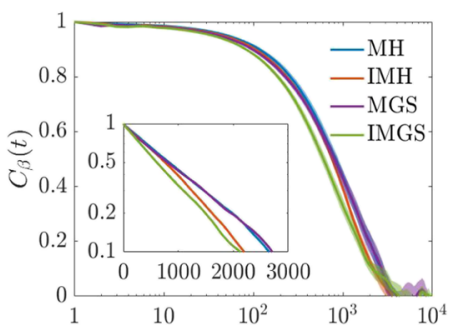

(i)

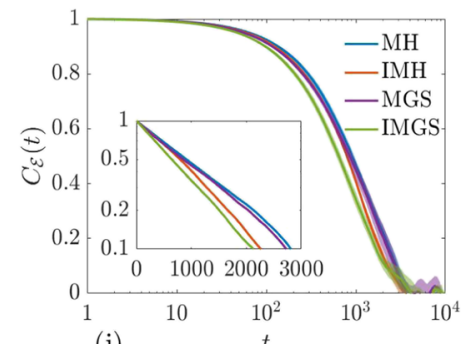

(j)

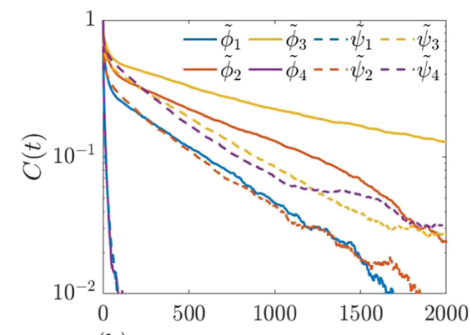

(k)

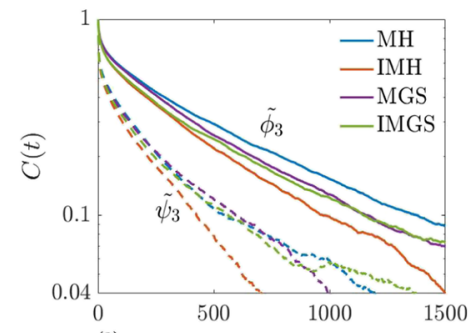

(1)

FIG. 6. Simulation results for ALA5 with $K=32$ temperatures. The deviation parameter $\delta=1$ for both IMH and IMGS. [(a)-(d)] Trajectories of inverse temperature $\beta$ and total energy $\mathscr{E}$ shown for the first $10^{5} \mathrm{MC}$ steps $t$. [(e)-(h)] Average free energy profile trajectories in $\phi_{3}$ at the lowest temperature; the shaded colors indicate the standard error on the average trajectory. The dashed line serves as a baseline comparison obtained from a much longer MD simulation with no simulated tempering ( 16.7 times longer simulation time). Units of free energy are in $\mathrm{kcal} / \mathrm{mol}$. [(i) and (j)] Average trajectory for the autocorrelation functions $C_{\beta}(t)$ and $C_{\mathscr{E}}(t)$; the shaded colors indicate standard error on the average trajectory. The insets show the same plots with the $y$-axis in the logarithmic scale. (k) Autocorrelation functions of dihedral angles (see the main text) obtained from a long MD simulation with no simulated tempering. (l) Autocorrelation functions of the slowest relaxing dihedral angles $\tilde{\phi}_{3}$ (solid lines) and $\tilde{\psi}_{3}$ (dashed lines) obtained from simulated tempering. 


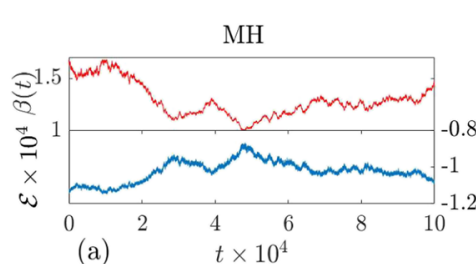

(a)
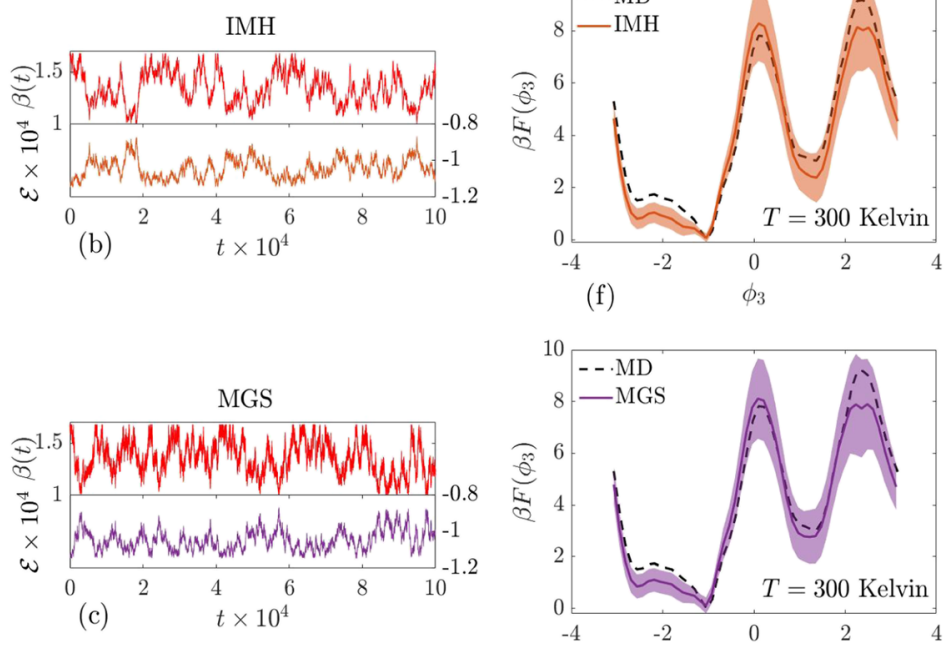

(g)

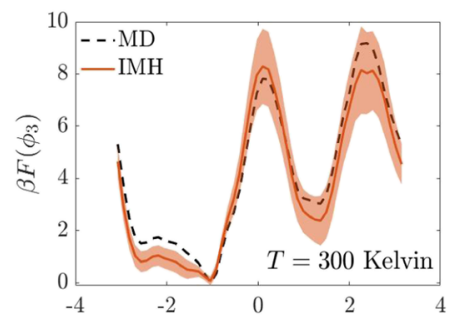

(f)

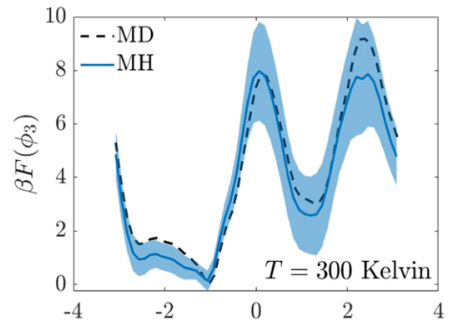

(e)

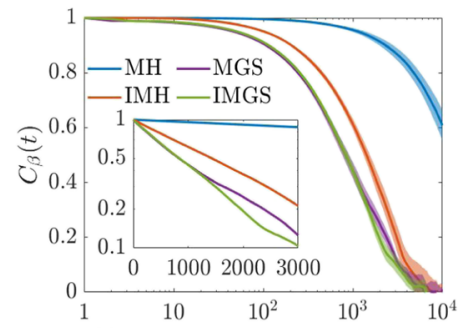

(i)

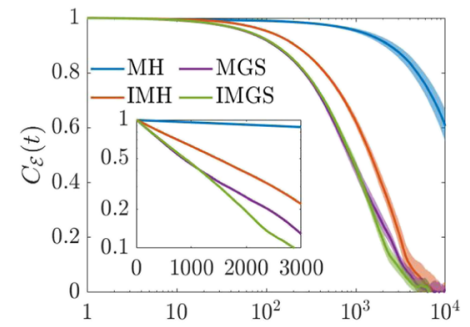

(j)

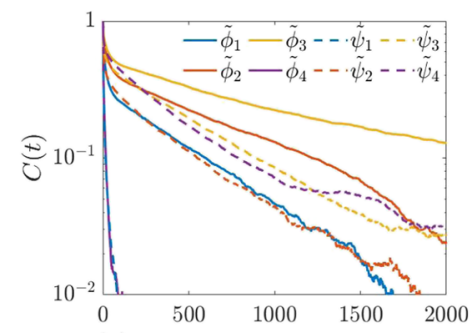

(k)

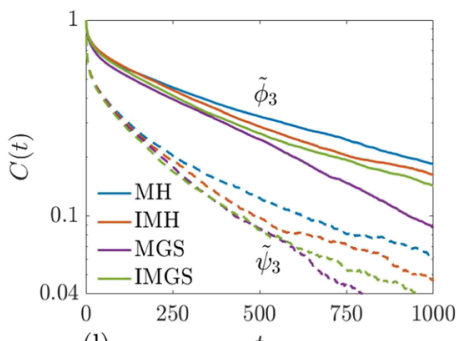

(1)

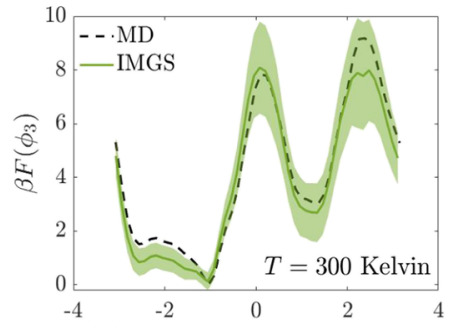

(h)

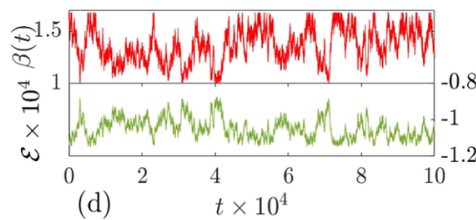

(d)

FIG. 7. Simulation results for ALA5 with $K=512$ temperatures. The deviation parameter $\delta=1$ for both IMH and IMGS. [(a)-(d)] Trajectories of inverse temperature $\beta$ and total energy $\mathscr{E}$ shown for the first $10^{5} \mathrm{MC}$ steps $t$. [(e)-(h)] Average free energy profile trajectories in $\phi_{3}$ at the lowest temperature; the shaded colors indicate the standard error on the average trajectory. The dashed line serves as a baseline comparison obtained from a much longer MD simulation with no simulated tempering ( 16.7 times longer simulation time). Units of free energy are in $\mathrm{kcal} / \mathrm{mol}$. [(i) and (j)] Average trajectory for the autocorrelation functions $C_{\beta}(t)$ and $C_{\mathscr{E}}(t)$; the shaded colors indicate standard error on the average trajectory. The insets show the same plots with the $y$-axis in the logarithmic scale. (k) Autocorrelation functions of dihedral angles (see the main text) obtained from a long MD simulation with no simulated tempering. (l) Autocorrelation functions of the slowest relaxing dihedral angles $\tilde{\phi}_{3}$ (solid lines) and $\tilde{\psi}_{3}$ (dashed lines) obtained from simulated tempering.

$K=32$, the trajectories of $\beta$ for all algorithms exhibit a random walk exploration of temperature space (Fig. 6, first column). Note that for IMH and IMGS, this observation is in contrast to that for the Ising model, where the IMH seemed to have a more deterministic exploration of temperature space and that of IMGS was ballistic. For both temperature domain sizes, we have plotted an average trajectory (obtained from six independent runs) for the autocorrelation functions of $\beta$ and total energy of the system $\mathscr{E}$. For $K=32$, the autocorrelation functions of $\beta$ and $\mathscr{E}$ suggest a modest gain for both IMH and IMGS over MH and MGS [Figs. 6(i) and 6(j)]. The corresponding integrated autocorrelation times $\tau_{\text {int }, \beta}$ and $\tau_{\text {int }, \mathscr{E}}$ recorded in Table I indicate a modest gain for the IMGS over MH. However, no statistically conclusive gain is observed over IMH and MGS. On the other hand, for a larger temperature domain size of $K=512$, 
TABLE I. The integrated autocorrelation times (in units of $\beta$ update trials $t$ ) for inverse temperature $\beta$, total energy $\mathscr{E}$, and slowest relaxing dihedral angles $\tilde{\phi}_{3}$ and $\tilde{\psi}_{3}$. The relative speed up for a given variable $f$ is defined with respect to the corresponding value $\tau_{\text {int }, f}$ of $\mathrm{MH}$ (see the work of Berendsen ${ }^{81}$ for error propagation on the relative speedup).

\begin{tabular}{|c|c|c|c|c|}
\hline & \multicolumn{4}{|c|}{ Integrated autocorrelation times $\left(\times 10^{3}\right)$} \\
\hline & $\tau_{\text {int }, \beta}$ & $\tau_{\text {int }, \mathscr{E}}$ & $\tau_{\text {int }, \tilde{\phi}_{3}}$ & $\tau_{\text {int }, \tilde{\psi}_{3}}$ \\
\hline & \multicolumn{4}{|c|}{$K=32$} \\
\hline $\mathrm{MH}$ & $2.4 \pm 0.2$ & $2.5 \pm 0.2$ & $1.3 \pm 0.4$ & $0.6 \pm 0.2$ \\
\hline IMH & $2.0 \pm 0.1$ & $2.06 \pm 0.09$ & $0.7 \pm 0.1$ & $0.29 \pm 0.03$ \\
\hline MGS & $2.3 \pm 0.2$ & $2.4 \pm 0.3$ & $0.8 \pm 0.1$ & $0.38 \pm 0.03$ \\
\hline \multirow[t]{2}{*}{ IMGS } & $2.0 \pm 0.2$ & $2.1 \pm 0.2$ & $0.9 \pm 0.3$ & $0.42 \pm 0.03$ \\
\hline & \multicolumn{4}{|c|}{$K=512$} \\
\hline $\mathrm{MH}$ & $33.0 \pm 4.0$ & $30.0 \pm 4.0$ & $1.9 \pm 0.7$ & $0.52 \pm 0.07$ \\
\hline IMH & $3.9 \pm 0.5$ & $3.9 \pm 0.4$ & $1.2 \pm 0.2$ & $0.44 \pm 0.09$ \\
\hline MGS & $2.9 \pm 0.2$ & $2.9 \pm 0.2$ & $0.67 \pm 0.09$ & $0.30 \pm 0.03$ \\
\hline IMGS & $2.6 \pm 0.2$ & $2.6 \pm 0.2$ & $1.0 \pm 0.2$ & $0.35 \pm \pm 0.08$ \\
\hline \multicolumn{5}{|c|}{ Relative speedup } \\
\hline & \multicolumn{4}{|c|}{$\mathrm{K}=32$} \\
\hline $\mathrm{MH}$ & 1.0 & 1.0 & 1.0 & 1.0 \\
\hline $\mathrm{IMH}$ & $1.2 \pm 0.1$ & $1.2 \pm 0.1$ & $1.9 \pm 0.6$ & $2.1 \pm 0.7$ \\
\hline MGS & $1.0 \pm 0.1$ & $1.0 \pm 0.2$ & $1.6 \pm 0.5$ & $1.6 \pm 0.5$ \\
\hline \multirow[t]{2}{*}{ IMGS } & $1.2 \pm 0.2$ & $1.2 \pm 0.1$ & $1.4 \pm 0.7$ & $1.4 \pm 0.5$ \\
\hline & \multicolumn{4}{|c|}{$K=512$} \\
\hline $\mathrm{MH}$ & 1.0 & 1.0 & 1.0 & 1.0 \\
\hline IMH & $8.5 \pm 1.5$ & $7.7 \pm 1.3$ & $1.6 \pm 0.6$ & $1.2 \pm 0.3$ \\
\hline MGS & $11.4 \pm 1.6$ & $10.3 \pm 1.6$ & $2.8 \pm 1.1$ & $1.7 \pm 0.3$ \\
\hline IMGS & $12.7 \pm 1.8$ & $11.5 \pm 1.8$ & $1.9 \pm 0.8$ & $1.5 \pm 0.4$ \\
\hline
\end{tabular}

notice from the trajectories of $\beta$ (Fig. 7, first column) that the mixing rate of $\beta$ and $\mathscr{E}$ for MH is drastically poor compared to the other three algorithms. Examining the corresponding integrated autocorrelation times in Table I, we notice that IMGS returns smaller integrated autocorrelation times for $\beta$ and $\mathscr{E}$ than both MH and IMH; however, compared to MGS, yet again, no conclusive gain is observed. It therefore seems that concerning the mixing times of $\beta$ and $\mathscr{E}$, the superiority of IMGS over IMH becomes more distinct in large temperature domain sizes. This is particularly clear when we compare the autocorrelation functions for $K=32$ [Figs. 6(i) and 6(j)] to those for $K=512$ [Figs. $7(\mathrm{i})$ and $7(\mathrm{j})$ ].

The dihedral angles for ALA5 are considered the slowest relaxing variables of the system. We had determined the slowest relaxing dihedral angles $(\phi, \psi)$ from a very long free MD simulation with no simulated tempering [Fig. $6(\mathrm{k})$ ]. To demonstrate convergence to the correct target distribution, we have constructed the free energy profiles in the slowest relaxing dihedral angle $\phi_{3}$ at the lowest temperature of $300 \mathrm{~K}$ (Figs. 6 and 7, second column). The trajectories shown are the average of six independent runs, each of which is plotted by re-weighting profiles at all temperatures with respect to the coldest temperature. As a baseline for comparison, we have used the free energy profile that is constructed using a very long free MD simulation. The baseline trajectory (shown in dashed line) consists of $~ 16.7$ times longer MD simulation time than the trajectories constructed using simulated tempering. In Fig. 6(1), we show the autocorrelation functions corresponding to the slowest dihedral angles, $\phi_{3}$ : C3-N4-CA4-C4 and $\psi_{3}$ : N3-CA3-C3-N4, obtained from simulated tempering simulations. Due to the circular nature of the dihedral coordinates, we have chosen to define the sinusoidal functions $\tilde{\phi}_{3}=1 / 2\left(\cos \phi_{3}+\sin \phi_{3}\right)$ and $\tilde{\psi}_{3}=1 / 2\left(\cos \psi_{3}+\sin \psi_{3}\right)$ and have plotted in Fig. 6(1) the average autocorrelation functions $C_{\tilde{\phi}_{3}}(t)$ and $C_{\tilde{\psi}_{3}}(t)$ obtained from six independent runs. To quantify the relaxation dynamics of the dihedral angles $\tilde{\phi}_{3}$ and $\tilde{\psi}_{3}$, we have computed the corresponding integrated autocorrelation times in Table I. Keeping in mind the standard error on the mean values, we observe a modest gain with IMGS over MH in both temperature domain sizes. However, no statistically conclusive gain is observed over IMH and MGS.

\section{DISCUSSION}

In this paper, we have generalized our recently introduced irreversible Gibbs sampler (IGS) and its variant the irreversible Metropolized-Gibbs sampler (IMGS) ${ }^{36}$ to the simulated tempering method. In particular, the IGS and IMGS, which break the DBC but satisfy the SDBC, are adapted for the update scheme of inverse temperature $\beta$ for a fixed configuration $\sigma$. We tested the correctness of our methods on a simple system described by a $1 \mathrm{D}$ model potential, whose exact weight factors $\omega(\beta)$ can be numerically computed. With this simple system, we demonstrated that our methods provide a significant improvement in the relaxation dynamics of inverse temperature and some system observables over the conventionally used simulated tempering with the Metropolis-Hastings scheme. When compared to the irreversible Metropolis-Hastings (IMH) method of Sakai and Hukushima, ${ }^{30}$ we observe that the improvement in the mixing time of $\beta$ and system observables accelerates with the increasing temperature domain size $K$.

Furthermore, we tested our methods on the Ising model and have shown that both the IGS and IMGS provide a decisive gain in the relaxation dynamics of $\beta$, magnetic susceptibility $\chi$, and energy density $\mathscr{E}$ by as much as 3.3 times when compared to their reversible counterparts with the DBC, respectively, the Gibbs sampler, and the Metropolized-Gibbs sampler. We demonstrated further that in both samplers, the furthest deviation from the DBC produces the shortest mixing time for $\beta, \chi$, and $\mathscr{E}$. For this system too, we provided comparison with $\mathrm{MH}$ and $\mathrm{IMH}$. The integrated autocorrelation times $\tau_{\text {int }, \beta}, \tau_{\text {int }, \chi}, \tau_{\text {int, } \mathscr{E}}$ for our methods scale (with respect to $K$ ) on the order of $\mathscr{O}(1)$. We compare this to $\mathrm{MH}$ and IMH, which scale on the order of $\mathscr{O}\left(K^{2}\right)$ and $\mathscr{O}(K)$, respectively. Our methods outperform the conventionally used $\mathrm{MH}$ for all domain sizes $K$. The gain in relaxation times over IMH is modest for small $K$ values but accelerates with increasing $K$. In summary, when compared to their respective reversible counterparts, our methods seem to provide a near fixed numerical gain in relaxation times at all temperature domain 
sizes $K$. However, the dynamical scaling of the integrated autocorrelation times suggests that the gain in sampling efficiency over both $\mathrm{MH}$ and $\mathrm{IMH}$ increases with increasing $K$.

We also performed simulated tempering MD simulations on alanine pentapeptide (ALA5) with temperature domain sizes, $K=32$ and $K=512$, equally spaced between $300 \mathrm{~K}$ and $500 \mathrm{~K}$. Guided by the performance on previous two systems, we chose to test the best of our two methods, namely, the IMGS, to compare performance with the existing methods. For $K=32$, the relaxation times of $\beta$ and total energy $\mathscr{E}$ indicate modest improvements for the IMGS over $\mathrm{MH}$, but no conclusive gain over IMH and MGS. However, at a larger temperature domain size, $K=512$, the IMGS provides a distinct improvement in the relaxation times of $\beta$ and $\mathscr{E}$ over both $\mathrm{MH}$ and $\mathrm{IMH}$, but no conclusive gain is observed over its reversible counterpart MGS. This is typical of both $\mathrm{MH}$ and $\mathrm{IMH}$, which perform optimally with nearest neighbor temperature swaps, and therefore, with increasing temperature domain size, one expects a less efficient sampling of the temperature space. For example, the integrated autocorrelation time of $\beta$ for $\mathrm{MH}$ algorithm scales on the order of $\mathscr{O}\left(K^{2}\right)$, as is expected of a random walk on domains of increasing size, while that of IMH scales on the order of $\mathscr{O}(K)$, as shown by Sakai and Hukushima ${ }^{30}$ for the Ising model and reproduced in this paper. The IMGS and its reversible counterpart, the MGS, however, are not restricted to nearest neighbor temperature swaps and perform a more global exploration of temperature space that provides a better mixing rate.

The relaxation dynamics of the slowest relaxing dihedral angles $\phi_{3}: \mathrm{C} 3-\mathrm{N} 4-\mathrm{CA} 4-\mathrm{C} 4$ and $\psi_{3}: \mathrm{N} 3-\mathrm{CA} 3-\mathrm{C} 3-\mathrm{N} 4$ indicate that for all three algorithms, IMH, IMGS, and MGS, $\phi_{3}$ and $\psi_{3}$ relax faster than with the conventionally used $\mathrm{MH}$ algorithm. However, the integrated autocorrelation times of the dihedral angles indicate that the IMGS performs fairly similarly to IMH and MGS, with no statistically conclusive gain over either method in both temperature domains $(K=32$ and $K=512)$. The slowest relaxing dihedral angles therefore do not distinguish the performance of our method from IMH and MGS with statistical significance.

A reason for this could be that in conventional simulated tempering, the relaxation rate of a given variable, say the magnetization of the system in the Ising model, cannot be slower than that at the coldest temperature, and equally, it cannot be faster than that at the hottest temperature. The slowest relaxation time of the system in simulated tempering therefore lies somewhere between those at the coldest and hottest temperatures. This follows from the work of Rosta and Hummer ${ }^{37}$ who had derived an expression for the maximum efficiency gain in simulated tempering simulations with ideally fast mixing rates. We consider the Ising model due to its relative simplicity and ease of generating large amounts of data. We show in Fig. 8 the theoretical prediction (black dashed line) from Ref. 37 for the optimum autocorrelation function of the magnetization density in our simulated tempering simulations. Also shown are the autocorrelation functions at the coldest (blue dashed line) and hottest (red dashed line) temperatures of the set obtained from very long free $\mathrm{MC}$ simulations with no simulated tempering and those obtained from simulated tempering with $\mathrm{MH}$ (blue solid line), IMH (orange solid line), and IMGS (green solid line). Note that the theoretical prediction lies between those of the coldest and hottest temperatures and the autocorrelation from the IMGS lies closest to the theoretically predicted optimum function. We wish to point out with this example that likewise, for simulated tempering with ALA5, there may exist an optimum efficiency gain concerning the relaxation time of the slowest dihedral angles. It is then feasible that the mixing time of the dihedral angles obtained from all algorithms are relatively close to the optimum predicted value, and therefore, the other three algorithms (IMH, MGS, and IMGS), which have demonstrated substantial gains over $\mathrm{MH}$ in the Ising model, now seem to produce only modest gains over $\mathrm{MH}$. Alternatively, the modest speedup could also be because, for some systems, it is possible that varying the temperature may not be the optimal collective variable to observe a speedup, and a Hamiltonian-based simulated tempering ${ }^{52-57}$ may be more suitable with a better chosen collective variable.

An extensive literature exists on techniques for enhancing the sampling efficiency of simulated and parallel tempering simulations. Among several studies, proposals have been made on determining the optimal temperature spacing, ${ }^{59-61}$ frequency of temperature exchange attempts, ${ }^{62,63}$ and optimum range and number of temperatures. ${ }^{37,58}$ In this paper, we introduced two irreversible methods for temperature swaps that are essentially based on Gibbs
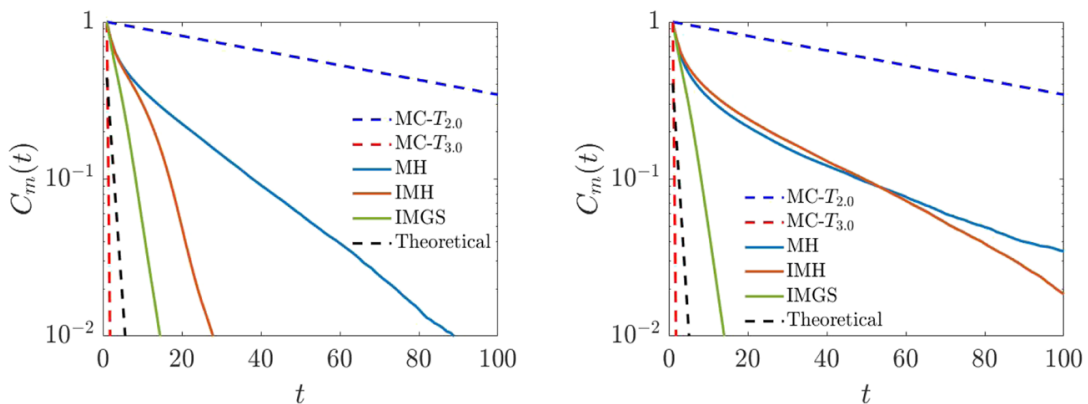

FIG. 8. Autocorrelation functions of the magnetization density in the $12 \times 12$ Ising model for $K=32$ (left) and $K=512$ (right) inverse temperatures between $\beta_{1}=0.5$ ( $T=$ $2.0)$ and $\beta_{K}=0.33(T=3.0)$ all equally spaced. The blue and red dashed lines are the autocorrelation functions obtained from a very long MC simulations at $\beta_{1}=0.5$ and $\beta_{K}=0.33$, respectively, with no simulated tempering. The solid lines are those obtained from simulated tempering simulations. The black dashed line is the theoretically predicted optimum value derived by Rosta and Hummer. ${ }^{37}$ The simulation parameters are $\Gamma=10^{2}$ sweeps and $\mathscr{T}=10^{7} \mathrm{MC}$ steps. The deviation parameter $\delta=1$ for both IMH and IMGS 
sampling techniques. For fair comparison to the widely used $\mathrm{MH}$ scheme and other existing methods, we therefore kept other important aspects of the simulation such as temperature spacing and range, number of temperatures, and frequency of temperature swaps constant across the different algorithms. It is of interest for a further study to also explore some of these aspects for our irreversible methods.

Conventional simulated tempering methods with the $\mathrm{MH}$ scheme perform optimally with nearest neighbor temperature swaps so that the temperature change may be accepted with a reasonable probability. Given a fixed temperature range, the number of temperatures $K$ needed scales as $\mathscr{O}\left(\mathscr{N}^{1 / 2}\right)^{15,53}$ for a system with degrees of freedom $\mathscr{N}$. While the choice of $K=512$ is practically unnecessary in our application to ALA5, for large complex systems, however, such as biologically relevant proteins consisting of hundreds of residues, a large number of temperatures $K$ are required to ensure optimum acceptance probability for temperature swaps. ${ }^{77}$ It is particularly for the simulation of large systems that conventional simulated tempering with the $\mathrm{MH}$ scheme proves to be inefficient at exploring temperature space, as is expected for a random walk in domains of increasing size. Given the robustness of our methods in an even excessively large temperature domain sizes, it is therefore hoped that our irreversible methods would provide a more efficient alternative to conventional methods for the simulation of large complex systems, such as biologically relevant proteins.

Although Sakai and Hukushima ${ }^{30}$ had shown with the Ising model that the IMH algorithm provides a square root reduction in the mixing time of inverse temperature as compared to $\mathrm{MH}$, our study here on the Ising model demonstrates that the mixing times with Gibbs sampling techniques including our irreversible methods scale on the order of $\mathscr{O}(1)$. In addition, our methods provide a further numerical gain ( 3.3 times in the case of a $25 \times 25$ Ising model) in relaxation times over the standard reversible Gibbs sampling techniques. Breaking the DBC can therefore pay off.

Another interesting and important aspect of classical spin systems is the study of the critical phenomena at phase transitions. Classical spin systems such as the Ising model suffer from a critical slowdown ${ }^{4}$ near the phase transition. The integrated autocorrelation times scale as $\tau_{\text {int }} \sim L^{z}$ at the critical temperature $T_{c}$, where $L$ is the linear dimensions of the system and $z$ here denotes the dynamical critical exponent. Often the scaling of integrated autocorrelation time with the system size is studied for the slowest relaxing observables of spin systems, such as magnetic susceptibility and energy, ${ }^{31,32}$ whose autocorrelation times describe the mixing time of the underlying Markov chain. For conventional Metropolis-type algorithms that use single spin-flip dynamics, the dynamical critical exponent is compatible with $z \sim 2$ scaling. Spin cluster algorithms, ${ }^{68,69}$ however, have been impressively superior to the study of critical phenomena when compared to conventional algorithms with single spin-flip dynamics. For example, for the $2 \mathrm{D} \mathrm{Ising}^{4}$ and $\mathrm{XY}$ model, ${ }^{31}$ the dynamical critical exponent is compatible with $z \sim 0$ scaling with Wolff's spin cluster algorithm ${ }^{69}$ when compared to $z$ $\sim 2$ scaling with the conventional Metropolis algorithm. However, the impressive performance of cluster algorithms remains limited to the application of a few spin models. Their performance remains particularly disappointing in application to more generic spin glass models in $3 \mathrm{D} .{ }^{70}$ On the other hand, simulation of spin glass models has been performed with conventional extended ensemble MCMC methods, such as the multicanonical method ${ }^{71-74}$ and simulated ${ }^{75,76}$ and parallel tempering. ${ }^{15}$ It remains of interest for a future study to explore the dynamical critical scaling of the integrated autocorrelation times with respect to the system size with our irreversible methods introduced here. Of particular interest is the computation of the dynamical critical exponent with our irreversible methods to compare with conventional simulated tempering with the DBC and spin cluster algorithms.

In summary, our methods do not only provide an efficiency gain over the conventionally used $\mathrm{MH}$ scheme in all practical temperature domain sizes but, particularly for simulations of large systems that may require large number of temperatures, can also be more efficient alternatives to both $\mathrm{MH}$ and $\mathrm{IMH}$, which suffer from dynamical scaling with respect to $K$. Further practical applications of our methods could extend to larger and/or more complex systems. In applications to ALA5 and biomolecular systems in general, it is worth investigating further if more distinct gains in sampling efficiency can be obtained with our methods using an alternative dynamical variable other than temperature in simulated tempering, for example, a dynamical variable in Hamiltonian-based simulated tempering that may be more effective than temperature in flattening the free energy landscape in $\phi / \psi$.

\section{SUPPLEMENTARY MATERIAL}

See the supplementary material for a flow chart of Algorithm 3.

\section{ACKNOWLEDGMENTS}

F.F. was supported by the EPSRC Center for Doctoral Training in Cross-Disciplinary Approaches to Non-Equilibrium Systems (EPSRC Reference No: EP/L015854/1). E.R. acknowledges support from the EPSRC (Grant No. EP/R013012/1) and the ERC (Project No. 757850 BioNet). The authors acknowledge the use of the research computing facility at King's College London, Rosalind (https://rosalind.kcl.ac.uk).

\section{NOMENCLATURE}

The transition probabilities from state $\left(\sigma, \beta_{k}\right)$ to $\left(\sigma, \beta_{l}\right)$ and commonly used abbreviations in the main text

$\begin{array}{ll}T\left(\boldsymbol{\sigma}, \beta_{l} \mid \boldsymbol{\sigma}, \beta_{k}\right) & \text { Generic transition probability } \\ G\left(\boldsymbol{\sigma}, \beta_{l} \mid \boldsymbol{\sigma}, \beta_{k}\right) & \text { Gibbs sampler (GS) } \\ M\left(\boldsymbol{\sigma}, \beta_{l} \mid \boldsymbol{\sigma}, \beta_{k}\right) & \text { Metropolized-Gibbs sampler (MGS) } \\ \mathscr{G}\left(\boldsymbol{\sigma}, \beta_{l} \mid \boldsymbol{\sigma}, \beta_{k}\right) & \text { Irreversible Gibbs sampler (IGS) } \\ \mathscr{M}\left(\boldsymbol{\sigma}, \beta_{l} \mid \boldsymbol{\sigma}, \beta_{k}\right) & \text { Irreversible Metropolized-Gibbs sampler (IMGS) } \\ \text { DBC } & \text { Detailed balance condition } \\ \text { BC } & \text { Balance condition } \\ \text { SDBC } & \text { Skewed detailed balance condition } \\ \text { MC } & \text { Monte Carlo } \\ \text { MCMC } & \text { Markov chain Monte Carlo } \\ \text { MH } & \text { Metropolis-Hastings } \\ \text { IMH } & \text { Irreversible Metropolis-Hastings }\end{array}$




\section{APPENDIX: THE CONDITIONAL $\tilde{\pi}(\beta, \varepsilon \mid \sigma)$ SATISFIES THE BALANCE CONDITION}

Here, we show that the conditional $\tilde{\pi}(\beta, \varepsilon \mid \boldsymbol{\sigma})$ satisfies the balance condition. We do this by writing the balance condition explicitly,

$$
\begin{aligned}
\tilde{\pi}(\beta, \varepsilon \mid \boldsymbol{\sigma})= & \sum_{\varepsilon^{\prime}} \sum_{\beta^{\prime}} \tilde{\pi}\left(\beta^{\prime}, \varepsilon^{\prime} \mid \boldsymbol{\sigma}\right) \mathscr{G}\left(\beta, \varepsilon, \boldsymbol{\sigma} \mid \beta^{\prime}, \varepsilon^{\prime}, \boldsymbol{\sigma}\right) \\
= & \sum_{\varepsilon^{\prime}} \sum_{\beta^{\prime} \neq \beta} \tilde{\pi}\left(\beta^{\prime}, \varepsilon^{\prime} \mid \boldsymbol{\sigma}\right) \mathscr{G}\left(\beta, \varepsilon, \boldsymbol{\sigma} \mid \beta^{\prime}, \varepsilon^{\prime}, \boldsymbol{\sigma}\right) \\
& +\sum_{\varepsilon^{\prime}} \sum_{\beta^{\prime}=\beta} \tilde{\pi}\left(\beta^{\prime}, \varepsilon^{\prime} \mid \boldsymbol{\sigma}\right) \mathscr{G}\left(\beta, \varepsilon, \boldsymbol{\sigma} \mid \beta^{\prime}, \varepsilon^{\prime}, \boldsymbol{\sigma}\right) .
\end{aligned}
$$

The first term on the right-hand side decomposes to

$$
\sum_{\beta^{\prime} \neq \beta} \tilde{\pi}\left(\beta^{\prime}, \varepsilon \mid \boldsymbol{\sigma}\right) \mathscr{G}\left(\beta, \varepsilon, \boldsymbol{\sigma} \mid \beta^{\prime}, \varepsilon, \boldsymbol{\sigma}\right)
$$

since $\mathscr{G}\left(\beta, \varepsilon, \boldsymbol{\sigma} \mid \beta^{\prime},-\varepsilon, \boldsymbol{\sigma}\right)=0, \quad \forall \beta^{\prime} \neq \beta$. The second term on the right-hand side of (A1) breaks down to

$$
\begin{aligned}
& \tilde{\pi}(\beta, \varepsilon \mid \boldsymbol{\sigma}) \mathscr{G}(\beta, \varepsilon, \boldsymbol{\sigma} \mid \beta, \varepsilon, \boldsymbol{\sigma})+\tilde{\pi}(\beta,-\varepsilon \mid \boldsymbol{\sigma}) \mathscr{G}(\beta, \varepsilon, \boldsymbol{\sigma} \mid \beta,-\varepsilon, \boldsymbol{\sigma}) \\
&=\tilde{\pi}(\beta, \varepsilon \mid \boldsymbol{\sigma}) \gamma^{(\varepsilon)}\left[1-\frac{1}{\gamma^{(\varepsilon)}} \Lambda(\beta,-\varepsilon, \boldsymbol{\sigma} \mid \beta, \varepsilon, \boldsymbol{\sigma})\right] \\
&+\tilde{\pi}(\beta,-\varepsilon \mid \boldsymbol{\sigma}) \gamma^{(-\varepsilon)}\left[\frac{1}{\gamma^{(-\varepsilon)}} \Lambda(\beta, \varepsilon, \boldsymbol{\sigma} \mid \beta,-\varepsilon, \boldsymbol{\sigma})\right] \\
&=\tilde{\pi}(\beta, \varepsilon \mid \boldsymbol{\sigma}) \gamma^{(\varepsilon)}-\tilde{\pi}(\beta, \varepsilon \mid \boldsymbol{\sigma}) \Lambda(\beta,-\varepsilon, \boldsymbol{\sigma} \mid \beta, \varepsilon, \boldsymbol{\sigma}) \\
&++\tilde{\pi}(\beta,-\varepsilon \mid \boldsymbol{\sigma}) \Lambda(\beta, \varepsilon, \boldsymbol{\sigma} \mid \beta,-\varepsilon, \boldsymbol{\sigma}),
\end{aligned}
$$

where $\gamma^{(\varepsilon)}=1-\sum_{\beta^{\prime} \neq \beta} \mathscr{G}\left(\beta^{\prime}, \varepsilon, \boldsymbol{\sigma} \mid \beta, \varepsilon, \boldsymbol{\sigma}\right)$. We therefore combine (A2) and (A3) to write

$$
\begin{aligned}
\tilde{\pi}(\beta, \varepsilon \mid \boldsymbol{\sigma})= & \sum_{\beta^{\prime}} \pi\left(\beta^{\prime}, \varepsilon \mid \boldsymbol{\sigma}\right) \mathscr{G}\left(\beta, \varepsilon, \boldsymbol{\sigma} \mid \beta^{\prime}, \varepsilon, \boldsymbol{\sigma}\right) \\
& -\tilde{\pi}(\beta, \varepsilon, \mid \boldsymbol{\sigma}) \Lambda(\beta,-\varepsilon, \boldsymbol{\sigma} \mid \beta, \varepsilon, \boldsymbol{\sigma}) \\
& +\tilde{\pi}(\beta,-\varepsilon \mid \boldsymbol{\sigma}) \Lambda(\beta, \varepsilon, \boldsymbol{\sigma} \mid \beta,-\varepsilon, \boldsymbol{\sigma}) \\
= & \sum_{\beta^{\prime}} \tilde{\pi}(\beta, \varepsilon \mid \boldsymbol{\sigma}) \mathscr{G}\left(\beta^{\prime}, \varepsilon, \boldsymbol{\sigma} \mid \beta, \varepsilon, \boldsymbol{\sigma}\right) \\
= & \tilde{\pi}(\beta, \varepsilon \mid \boldsymbol{\sigma}) \\
= & \frac{1}{2} \pi(\beta \mid \boldsymbol{\sigma}),
\end{aligned}
$$

where we have obtained the second equality by invoking the balance condition in (20).

\section{DATA AVAILABILITY}

The data that support the findings of this study are available from the corresponding author upon reasonable request.

\section{REFERENCES}

${ }^{1}$ N. Metropolis, A. W. Rosenbluth, M. N. Rosenbluth, A. H. Teller, and E. Teller, "Equation of state calculations by fast computing machines," J. Chem. Phys. 21, 1087 (1953).

${ }^{2}$ W. K. Hastings, "Monte Carlo sampling methods using Markov chains and their applications," Biometrika 57, 97-109 (1970).

${ }^{3}$ D. P. Landau and K. Binder, A Guide to Monte Carlo Simulations in Statistical Physics, 2nd ed. (Cambridge University Press, Cambridge, 2005).

${ }^{4}$ M. E. J. Newman and G. T. Barkema, Monte Carlo Methods in Statistical Physics (Oxford University Press, New York, 2001).

${ }^{5}$ U. H. E. Hansmann and Y. Okamoto, "New Monte Carlo algorithms for protein folding," Curr. Opin. Struct. Biol. 9, 177-183 (1999).

${ }^{6}$ A. Kolinski and J. Skolnick, "Monte Carlo simulations of protein folding. II. Application to protein A, ROP, and crambin," Proteins: Struct., Funct., Genet. 18, 353-366 (1994).

${ }^{7}$ R. W. Shonkwiler, Finance with Monte Carlo (Springer, New York, 2013).

${ }^{8}$ S. Geman and D. Geman, "Stochastic relaxation, Gibbs distributions, and the Bayesian restoration of images," IEEE Trans. Pattern Anal. Mach. Intell. PAMI-6, 721-741 (1984).

${ }^{9}$ A. Mitsutake, Y. Sugita, and Y. Okamoto, "Generalized-ensemble algorithms for molecular simulations of biopolymers," Biopolymers 60, 96-123 (2001).

${ }^{10}$ B. A. Berg and T. Neuhaus, "Multicanonical algorithms for first order phase transitions," Phys. Lett. B 267, 249-253 (1991).

${ }^{11}$ B. A. Berg and T. Neuhaus, "Multicanonical ensemble: A new approach to simulate first-order phase transitions," Phys. Rev. Lett. 68, 9-12 (1992).

${ }^{12}$ E. Marinari and G. Parisi, "Simulated tempering: A new Monte Carlo scheme," Europhys. Lett. 19, 451-458 (1992).

${ }^{13}$ R. H. Swendsen and J.-S. Wang, "Replica Monte Carlo simulation of spinglasses," Phys. Rev. Lett. 57, 2607 (1986).

${ }^{14}$ C. J. Geyer, "Markov chain Monte Carlo maximum likelihood," in Computing Science and Statistics: Proceedings of the 23rd Symposium on the Interface, Seattle, Washington, April 21-24, 1991 (Interface Foundation of North America, New York, 1991), pp. 156-163.

${ }^{15} \mathrm{~K}$. Hukushima and K. Nemoto, "Exchange Monte Carlo method and application to spin glass simulations," J. Phys. Soc. Jpn. 65, 1604-1608 (1996).

${ }^{16}$ U. H. E. Hansmann, "Parallel tempering algorithm for conformational studies of biological molecules," Chem. Phys. Lett. 281, 140-150 (1997).

${ }^{17}$ Y. Sugita and Y. Okamoto, "Replica-exchange molecular dynamics method for protein folding," Chem. Phys. Lett. 314, 141-151 (1999).

${ }^{18}$ L. Tierney, "Markov chains for exploring posterior distributions," Ann. Stat. 22, 1701-1728 (1994).

${ }^{19}$ S. P. Meyn and R. L. Tweedie, Markov Chains and Stochastic Stability (SpringerVerlag, London, 1993).

${ }^{20}$ V. I. Manousiouthakis and M. W. Deem, "Strict detailed balance is unnecessary in Monte Carlo simulation," J. Chem. Phys. 110, 2753 (1999).

${ }^{21}$ P. Diaconis, S. Holmes, and R. M. Neal, "Analysis of a nonreversible Markov chain sampler," Ann. Appl. Probab. 10, 726-752 (2000).

${ }^{22}$ F. Chen, L. Lovász, and I. Pak, "Lifting Markov chains to speed up mixing," in Proceedings of the 31st Annual ACM Symposium on Theory of Computing, Atlanta, GA, USA, May 1-4, 1999 (Association for Computing Machinery, NY, USA, 1999), pp. 275-281.

${ }^{23}$ R. D. Schram and G. T. Barkema, "Monte Carlo methods beyond detailed balance," Physica A 418, 88-93 (2015).

${ }^{24} \mathrm{H}$. Suwa and S. Todo, "Markov chain Monte Carlo method without detailed balance," Phys. Rev. Lett. 105, 120603 (2010).

${ }^{25}$ K. S. Turitsyn, M. Chertkov, and M. Vucelja, "Irreversible Monte Carlo algorithms for efficient sampling," Physica D 240, 410-414 (2011). 
${ }^{26} \mathrm{H}$. C. M. Fernandes and M. Weigel, "Non-reversible Monte Carlo simulations of spin models," Comput. Phys. Commun. 182, 1856-1859 (2011).

${ }^{27}$ Y. Sakai and K. Hukushima, "Dynamics of one-dimensional Ising model without detailed balance condition," J. Phys. Soc. Jpn. 82, 064003 (2013).

${ }^{28} \mathrm{~K}$. Hukushima and Y. Sakai, "An irreversible Markov-chain Monte Carlo method with skew detailed balance conditions," J. Phys.: Conf. Ser. 473, 012012 (2013).

${ }^{29}$ Y. Sakai and K. Hukushima, "Eigenvalue analysis of an irreversible random walk with skew detailed balance conditions," Phys. Rev. E 93, 043318 (2016).

${ }^{30}$ Y. Sakai and K. Hukushima, "Irreversible simulated tempering," J. Phys. Soc. Jpn. 85, 104002 (2016).

${ }^{31}$ M. Michel, J. Mayer, and W. Krauth, "Event-chain Monte Carlo for classical continuous spin models," Europhys. Lett. 112, 20003 (2015).

${ }^{32}$ Y. Nishikawa, M. Michel, W. Krauth, and K. Hukushima, "Event-chain algorithm for the Heisenberg model: Evidence for $z \simeq 1$ dynamic scaling," Phys. Rev. E 92, 063306 (2015)

${ }^{33}$ A. Ichiki and M. Ohzeki, "Violation of detailed balance accelerates relaxation," Phys. Rev. E 88, 020101 (2013).

${ }^{34}$ M. Kaiser, R. L. Jack, and J. Zimmer, "Acceleration of convergence to equilibrium in Markov chains by breaking detailed balance," J. Stat. Phys. 168, 259-287 (2017).

${ }^{35}$ J. S. Liu, "Peskun's theorem and a modified discrete-state Gibbs sampler," Biometrika 83, 681-682 (1996).

${ }^{36}$ F. Faizi, G. Deligiannidis, and E. Rosta, "Efficient irreversible Monte Carlo samplers," J. Chem. Theory Comput. 16, 2124-2138 (2020).

${ }^{37}$ E. Rosta and G. Hummer, "Error and efficiency of simulated tempering simulations," J. Chem. Phys. 132, 034102 (2010).

${ }^{38}$ J. D. Chodera and M. R. Shirts, "Replica exchange and expanded ensemble simulations as Gibbs sampling: Simple improvements for enhanced mixing," J. Chem. Phys. 135, 194110 (2011).

${ }^{39} \mathrm{Y}$. Mori and H. Okumura, "Simulated tempering based on global balance or detailed balance conditions: Suwa-Todo, heat bath, and Metropolis algorithms," J. Comput. Chem. 36, 2344-2349 (2015).

${ }^{40}$ E. Marinari, G. Parisi, and J. Ruiz-Lorenzo, "Numerical simulations of spin glass systems," arXiv:cond-mat/9701016 [cond-mat.dis-nn] (1997); accessed 13 April 2020.

${ }^{41}$ A. Irbäck and F. Potthast, "Studies of an off-lattice model for protein folding: Sequence dependence and improved sampling at finite temperature," J. Chem. Phys. 103, 10298 (1995).

${ }^{42}$ U. H. E. Hansmann and Y. Okamoto, "Numerical comparisons of three recently proposed algorithms in the protein folding problem," J. Comput. Chem. 18, 920933 (1997).

${ }^{43}$ S. Park and V. S. Pande, "Choosing weights for simulated tempering," Phys. Rev. E 76, 016703 (2007).

${ }^{44}$ P. H. Nguyen, Y. Okamoto, and P. Derreumaux, "Communication: Simulated tempering with fast on-the-fly weight determination," J. Chem. Phys. 138, 061102 (2013).

${ }^{45} \mathrm{~A}$. Mitsutake and Y. Okamoto, "Replica-exchange simulated tempering method for simulations of frustrated systems," Chem. Phys. Lett. 332, 131-138 (2000).

${ }^{46}$ A. A. Barker, "Monte Carlo calculations of the radial distribution functions for a proton-electron plasma," Aust. J. Phys. 18, 119-134 (1965).

${ }^{47}$ L. Pollet, S. M. A. Rombouts, K. V. Houcke, and K. Heyde, "Optimal Monte Carlo updating," Phys. Rev. E 70, 056705 (2004).

${ }^{48}$ J. D. Chodera, W. C. Swope, J. W. Pitera, C. Seok, and K. A. Dill, "Use of the weighted histogram analysis method for the analysis of simulated and parallel tempering simulations," J. Chem. Theory Comput. 3, 26-41 (2006).

${ }^{49}$ S. Kumar, J. M. Rosenberg, D. Bouzida, R. H. Swendsen, and P. A. Kollman, "The weighted histogram analysis method for free-energy calculations on biomolecules. I. The method," J. Comput. Chem. 13, 1011-1021 (1992).

${ }^{50}$ R. Ren and G. Orkoulas, "Acceleration of Markov chain Monte Carlo simulations through sequential updating," J. Chem. Phys. 124, 064109 (2006).

${ }^{51}$ K. Huang, Statistical Mechanics, 2nd ed. (Wiley, New York, 1987).
${ }^{52}$ Y. Sugita, A. Kitao, and Y. Okamoto, “ Multidimensional replica-exchange method for free-energy calculations,” J. Chem. Phys. 113, 6042 (2000).

${ }^{53}$ H. Fukunishi, O. Watanabe, and S. Takada, "On the Hamiltonian replica exchange method for efficient sampling of biomolecular systems: Application to protein structure prediction," J. Chem. Phys. 116, 9058 (2002).

${ }^{54}$ S. G. Itoh and H. Okumura, "Hamiltonian replica-permutation method and its applications to an alanine dipeptide and amyloid- $\beta(29-42)$ peptides," J. Comput. Chem. 34, 2493-2497 (2013).

${ }^{55} \mathrm{M}$. Meli and G. Colombo, "A Hamiltonian replica exchange molecular dynamics (MD) method for the study of folding, based on the analysis of the stabilization determinants of proteins," Int. J. Mol. Sci. 14, 12157-12169 (2013).

${ }^{56}$ J. Hritz and C. Oostenbrink, "Hamiltonian replica exchange molecular dynamics using soft-core interactions," J. Chem. Phys. 128, 144121 (2008).

${ }^{57}$ S. Jang, S. Shin, and Y. Pak, "Replica-exchange method using the generalized effective potential," Phys. Rev. Lett. 91, 058305 (2003).

${ }^{58} \mathrm{E}$. Rosta and G. Hummer, "Error and efficiency of replica exchange molecular dynamics simulation," J. Chem. Phys. 131, 165102 (2009).

${ }^{59}$ Y. F. Atchadé, G. O. Roberts, and J. S. Rosenthal, "Towards optimal scaling of metropolis-coupled Markov chain Monte Carlo," Stat. Comput. 21, 555-568 (2011).

${ }^{60}$ A. Kone and D. A. Kofke, "Selection of temperature intervals for paralleltempering simulations," J. Chem. Phys. 122, 206101 (2005).

${ }^{61}$ W. D. Vousden, W. M. Farr, and I. Mandel, "Dynamic temperature selection for parallel tempering in Markov chain Monte Carlo simulations," Mon. Not. R. Astron. Soc. 455, 1919-1937 (2016).

${ }^{62}$ D. Sindhikara, Y. Meng, and A. E. Roitberg, "Exchange frequency in replica exchange molecular dynamics," J. Chem. Phys. 128, 024103 (2008).

${ }^{63}$ M. J. Abraham and J. E. Gready, "Ensuring mixing efficiency of replicaexchange molecular dynamics simulations," J. Chem. Theory Comput. 4, 11191128 (2008).

${ }^{64}$ S. Jo, T. Kim, V. G. Iyer, and W. Im, "CHARMM-GUI: A web-based graphical user interface for CHARMM," J. Comput. Chem. 29, 1859-1865 (2008).

${ }^{65}$ J. C. Phillips, R. Braun, W. Wang, J. Gumbart, E. Tajkhorshid, E. Villa, C. Chipot, R. D. Skeel, L. Kalé, and K. Schulten, "Scalable molecular dynamics with NAMD," J. Comput. Chem. 26, 1781-1802 (2005).

${ }^{66}$ J. Huang, S. Rauscher, G. Nawrocki, T. Ran, M. Feig, B. L. de Groot, H. Grubmüller, and A. D. Mackerell, "CHARMM36m: An improved Force field for folded and intrinsically disordered proteins," Nat. Methods 14, 71-73 (2017).

${ }^{67}$ T. Darden, D. York, and L. Pedersen, "Particle mesh Ewald: An Nlog(N) method for Ewald sums in large systems," J. Chem. Phys. 98, 10089 (1993).

${ }^{68}$ R. H. Swendsen and J.-S. Wang, "Nonuniversal critical dynamics in Monte Carlo simulations," Phys. Rev. Lett. 58, 86 (1987).

${ }^{69} \mathrm{U}$. Wolff, "Collective Monte Carlo updating for spin systems," Phys. Rev. Lett. 62, 361 (1989).

${ }^{70}$ D. A. Kesler and M. Bretz, "Unbridled growth of spin-glass clusters," Phys. Rev. B 41, 4778 (1990).

${ }^{71}$ B. A. Berg and T. Celik, "New approach to spin-glass simulations," Phys. Rev. Lett. 69, 2292 (1992).

${ }^{72}$ B. A. Berg and T. Celik, "The multicanonical ensemble: A new approach to computer simulations," Int. J. Mod. Phys. C 03, 1083-1098 (1992).

${ }^{73}$ B. A. Berg, T. Celik, and U. Hansmann, "Multicanonical study of the 3D ising spin glass," Europhys. Lett. 22, 63 (1993).

${ }^{74}$ B. A. Berg, U. E. Hansmann, and T. Celik, "Ground-state properties of the threedimensional Ising spin glass," Phys. Rev. B 50, 16444 (1994).

${ }^{75} \mathrm{~W}$. Kerler and P. Rehberg, "Simulated-tempering procedure for spin-glass simulations," Phys. Rev. E 50, 4220 (1994).

${ }^{76}$ B. Coluzzi, "Numerical simulations on the 4 D Heisenberg spin glass," J. Phys. A: Math. Gen. 28, 747 (1995).

${ }^{77} \mathrm{~J}$. W. Pitera, I. Haque, and W. C. Swope, "Absence of reptation in the hightemperature folding of the trpzip $2 \beta$-hairpin peptide," J. Chem. Phys. 124, 141102 (2006). 
${ }^{78}$ J.-S. Wang and R. H. Swendsen, "Transition matrix Monte Carlo method," J. Stat. Phys. 106, 245-285 (2002).

${ }^{79}$ J.-S. Wang, T. K. Tay, and R. H. Swendsen, "Transition matrix Monte Carlo reweighting and dynamics," Phys. Rev. Lett. 82, 476 (1999).
${ }^{80}$ J.-S. Wang, "Transition matrix Monte Carlo method," Comput. Phys. Commun. 121-122, 22-25 (1999).

${ }^{81}$ H. J. C. Berendsen, A Student's Guide to Data and Error Analysis (Cambridge University Press, New York, 2011). 